\title{
ANÁLISIS COMPARATIVO DE TENDENCIAS EN LA PRECIPITACIÓN, POR DISTINTOS INPUTS, ENTRE LOS DOMINIOS HIDROLÓGICOS DEL SEGURA Y DEL JÚCAR
} (1958-2008)

\author{
Javier Miróa $^{\text {a }}$ María José Estrela ${ }^{\mathrm{b}}$, Francisco Pastor ${ }^{\mathrm{a}}$ y Millán Millán ${ }^{\mathrm{a}}$
}

\section{RESUMEN}

Recientemente han sido varios los trabajos que han analizado las tendencias en la precipitación dependiendo del tipo de flujo que la ha producido. Esta metodología novedosa ya se ha aplicado en el análisis de las precipitaciones en la Comunidad Valenciana, según tres tipos sinópticos básicos que las provocan: 1) precipitaciones convectivas, 2) de tipo frontal, y 3 ) las advecciones mediterráneas (Frente de Retroceso), detectándose tendencias significativas que no habrían sido detectadas con otro enfoque o metodología. El objetivo de este trabajo es ampliar este análisis mediante un estudio comparativo entre los dominios hidrográficos del Segura y del Júcar, y dentro de estos, los subdominios o sectores interiores frente a litorales. La utilización sólo de las series disponibles con 50 años, y de técnicas de análisis más fiables, nos va a permitir validar estas tendencias. Los resultados obtenidos muestran tendencias claras al descenso de la precipitación en el caso de las advecciones atlánticas, y ahora también en las del Norte (retroceso seca), significativas en el interior, y de menor peso o compensadas en el litoral. Además, se detectan paralelismos, junto con alguna diferencia, entre ambos dominios analizados.

Palabras clave: inputs de precipitación, tendencias, análisis territorial comparativo, frente de retroceso, desagregación de la precipitación.

\section{ABSTRACT}

Several studies have recently analized the trends in the precipitation depending on the type of meteorological conditions that provokes it. This new methodology has already been applied on the analisys of the precipitation in the Valencia Region. The types of precipitation are: a) convective, b) frontal systems, c) mediterranean advections. With this study we detect significant trends that would not have been working only with total

Laboratorio de Meteorología-Climatología, Unidad Mixta CEAM-UVEG. Fundación Centro de Estudios Ambientales del Mediterráneo, Área de Meteorología-Climatología, Parque Tecnológico, Calle Charles R. Darwin 14, 46980 Paterna, Valencia, Spain.

b Laboratorio de Meteorología-Climatología, Unidad Mixta CEAM-UVEG (Universidad de Valencia). Departament de Geografia Física, Universitat de Valencia, Avda. Blasco Ibáñez 28, 46010 Valencia, Spain. 
precipitations. The objective of this work is to expand this analisys to two near areas and compare results. We are working on the hidrographics basin of Segura and Jucar. The results show clear decreasing trends of the precipitation for the frontal system type and also for the advections from the North. There is similar behaviour between the two basins.

Key words: inputs of precipitation, trends, mediterranean advections (backdoor cold fronts), disaggregate the precipitation series.

\section{Introducción y objetivos}

Una revisión de la bibliografía sobre la precipitación estacional, anual, y sus tendencias tanto a escalas local como global, muestran importantes variaciones de un área a otra dentro del continente europeo. La mayoría de los trabajos hablan de una tendencia positiva en la precipitación para los países del Norte de Europa y, por el contrario, una tendencia claramente negativa para los países del Sur (Hougton et al, 1996 y 2001; Schönwiese y Rapp, 1997; Brizio y Mercalli, 1992; Quereda et al. 1996; Buffoni et al., 1998 y 1999; Brunetti et al. 2001). De hecho, estudios específicos en la Península Ibérica apuntan a reducciones de precipitación en la mitad sur, que son más acusadas hacia el sur y hacia el este (Sales et al., 1982; Martín Vide, 1987; Raso, 1996; Montón y Quereda, 1997). Otros estudios no muestran una tendencia tan marcada en la evolución de los totales de precipitación para España pero sí muestran una clara tendencia en la variabilidad interanual durante los últimos años del siglo XX (Almarza, 2002). Conclusiones similares se han derivado del análisis de series reconstruidas desde 1864 hasta la actualidad para el Este y sureste de España (Chazarra y Almarza, 2002).

Sin embargo, todos estos estudios tienen en común que trabajan con los totales de la precipitación, y generalmente no analizan sus cambios estructurales, al no contemplarse si las variaciones en los totales de precipitación pueden estar relacionadas con cambios en el tipo de evento, es decir, en las condiciones meteorológicas que las producen. Los autores de este trabajo comenzaron considerando que este tipo de análisis podría dar alguna explicación a la pérdida de tormentas de verano que miembros del equipo ya detectaron durante la preparación de las campañas de medida en Francia, Italia y España dentro del Proyecto MECAPIP (Millán y Artiñano, 1992). Por esta razón desarrollaron una técnica novedosa de desagregación de la precipitación según origen genético o meteorológico (Millán et al., 2005 a, b). Los resultados más destacables de estos trabajos, si bien confirman una tendencia a la pérdida de la precipitación total en algunas zonas de la Comunidad Valenciana, también revelan que esta generalización encubre diferencias importantes relacionadas con el tipo de precipitación, ya sean precipitaciones procedentes de sistemas frontales atlánticos, tormentas convectivas, o advecciones mediterráneas, también denominadas frentes de retroceso. Mientras que en el interior se detectó una disminución en los totales de precipitación de origen atlántico y de tormentas de verano, en las zonas costeras se detectó un aumento relativo de las cantidades procedentes de frentes de retroceso que han conllevado un aumento en los volúmenes así como de su irregularidad.

Con el interés por conocer si estas pautas en la precipitación se están detectando en otros sectores de la Península, con problemas en el recurso 'agua' similares a los existentes en la Comunidad Valenciana, estamos llevando a cabo análisis en áreas próximas a fin de comparar los resultados.

El objetivo del presente trabajo es aplicar el método de desagregación de la precipitación, según el origen genético que la provoca, al análisis comparativo de la precipitación de dos dominios hidrográficos, el Segura y el Júcar, y dentro de estos, a los subdominios interiores frente a los litorales. El análisis está basado en el método desarrollado por los autores (Millán 
et al., 2005a), aunque en este caso se ha mejorado el proceso de desagregación por inputs al automatizarlo, con el objeto de eliminar la parte de subjetividad que llevaba implícito, así como de facilitar la ampliación tanto espacial como temporal del análisis. El estudio abarca el período 1958-2008, aunque para determinados análisis se han diferenciado dos etapas (19581978 y 1988-2008), a fin de detectar cambios estructurales en la precipitación.

\section{Metodología}

\subsection{Obtención y catalogación de los inputs sobre una base diaria}

En primer lugar se ha determinado un método para la adscripción de todos los días disponibles desde 1958 hasta 2008, a uno de los tres inputs pluviométricos definidos. En anteriores trabajos de los autores (Estrela et al., 2004 y 2006; Millán et al., 2005 y 2006; Miró et al., 2006) ya se utilizó la diferenciación entre: 1) situaciones convectivas, 2) advección atlántica ó de tipo frontal, y 3 ) advección de retroceso (mediterránea), con la finalidad de diferenciar situaciones con el predominio de un tipo de flujo o advección en capas mediasbajas (sean ciclónicas o anticiclónicas). Ello permite diferenciar la precipitación según su lugar de recarga preferente, ya sea atlántico ó mediterráneo. Las precipitaciones de carácter convectivo están determinadas por el predominio de los regímenes de brisas en el semestre estival, baja térmica superficial en el interior de la Península o el Norte de África, y flujo discordante entre las brisas superficiales y el flujo observado en niveles medios y altos de la troposfera (Millán y Artiñano, 1992).

La laboriosidad de esta catalogación manual, junto al carácter subjetivo que tiene la asignación de la situación diaria a un tipo sinóptico específico, nos ha llevado al desarrollo de un método automatizado. Entre las ventajas del nuevo método, aparte de su mayor objetividad, cabe destacar su fácil ampliación tanto temporal como espacialmente. El método se basa en el uso de los datos de reanálisis del CDAS-NCEP/NCAR (http://nomad1.ncep.noaa.gov/ ncep_data/index.html), disponibles desde 1950 hasta la actualidad, dentro de una malla que comprende las latitudes entre $42,5^{\circ} \mathrm{N}$ y $35^{\circ} \mathrm{N}$ y las longitudes entre $5^{\circ} \mathrm{W}$ y $5^{\circ} \mathrm{E}$. La malla está compuesta por puntos a $2,5^{\circ}$ de distancia del vecino (Fig. 1). Básicamente se utiliza la capa de $925 \mathrm{hPa}$, determinando la dirección del flujo entre cada punto y sus vecinos, a las 12h UTC. Finalmente se suman todos los vectores, y el flujo predominante se adscribe automáticamente a un input, según las direcciones de flujo que se muestran en la Figura 1.

En este trabajo se ha incorporado un nuevo input ('retroceso seca'), que no estaba contemplado en los trabajos previos (Estrela et al., 2004 y 2006; Millán et al., 2005 y 2006; Miró et al., 2006), y que incluye los flujos de procedencia Nord-Noroeste, Norte y Nordeste sin apenas recorrido marítimo. Aunque consideramos que este nuevo input tendrá poco peso en el caso de la fachada mediterránea peninsular, su inclusión se debe a la necesidad de elaborar una tipología aplicable y válida para toda la cuenca del Mediterráneo, en su vertiente europea, que permita realizar comparaciones entre áreas. De hecho, en sectores como el Sur de Francia, Italia o Grecia, las advecciones directas desde el interior del continente europeo sí son importantes.

Por último, la delimitación de las situaciones convectivas ha requerido un cálculo más elaborado, basado en la coincidencia de varios factores, a las $12 \mathrm{~h}$ UTC. Estos son: a) la presencia de un flujo dominante discordante entre la capa de $700 \mathrm{hPa}$ (predominio de terrales o flujo muy débil) y la de $925 \mathrm{hPa}$ (flujo contrario); b) la presencia, a $925 \mathrm{hPa}$, de presiones relativamente más bajas en el conjunto de puntos de la malla sobre terreno continental que en el conjunto que está sobre el mar, diferencia que se diluye a 700hPa; y c) no existencia de un flujo dominante, en una dirección concreta generalizada en la ma- 


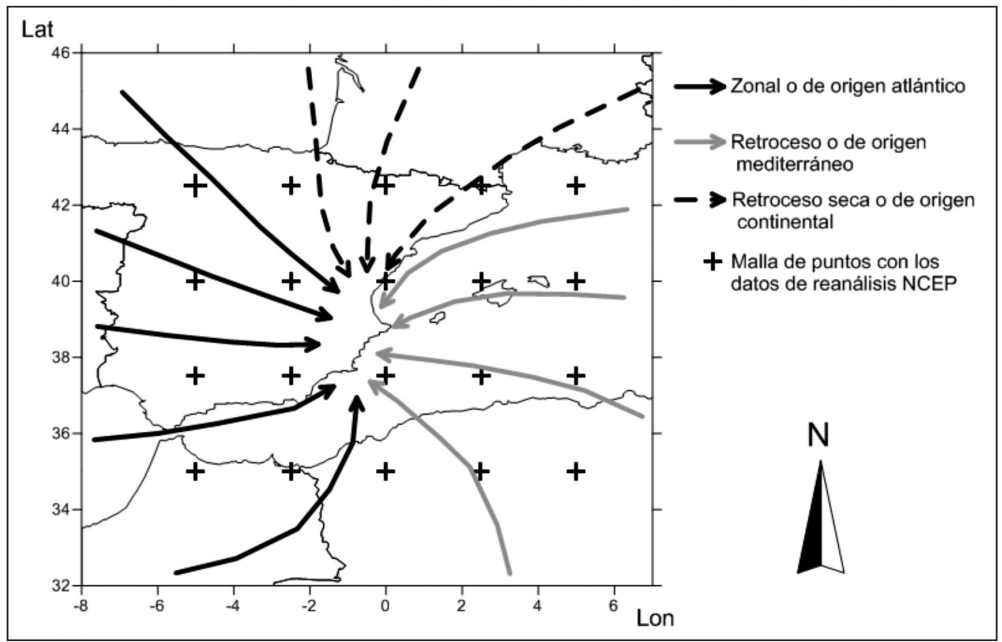

FIGURA 1. Flujos que componen cada input, y malla de datos de reanálisis del NCEP utilizada para el cálculo del flujo dominante.

lla, y de potencia por encima de un umbral. Baste decir que los resultados obtenidos con este método automático son muy similares a los alcanzados con anterioridad mediante la catalogación manual. El resultado final es una serie de base diaria, en la que cada día se adscribe a uno de los siguientes inputs: a) zonal o 'atlántico', b) 'retroceso' mediterráneo, c)'retroceso seca' o continental, y d) 'convectiva' (Fig. 1).

\subsection{Marco territorial y series de precipitación utilizadas}

Los dos ámbitos territoriales que se comparan están demarcados esencialmente por las confederaciones hidrográficas del Segura y la del Júcar. Cada uno de estos bloques hidrológicos se ha dividido a su vez en un sector interior, a más de $40 \mathrm{~km}$ de la costa, un sector prelitoral, entre 20 y $40 \mathrm{~km}$ de la costa, y un sector litoral a menos de $20 \mathrm{~km}$ de la costa. Esta división se hace necesaria porque la detección de tendencias en la precipitación es especialmente relevante hacia el interior, dado el déficit hídrico estructural que ya de por sí presentan tanto la cuenca del Segura como la del Júcar.

Se han seleccionado varias series de precipitación representativas de cada ámbito descrito, a partir de la red de estaciones pluviométricas de la Agencia Estatal de Meteorología (AEMET). El criterio principal de selección ha sido el que cubran el periodo 1958-2008 (51 años) con un porcentaje de datos válidos superior al $95 \%$ de dicho periodo. Según ámbitos, la distribución de estaciones es la siguiente: Segura interior 25 estaciones; Segura prelitoral 17 estaciones; Segura litoral 6 estaciones; Júcar interior 14 estaciones; Júcar prelitoral 12 estaciones; y Júcar litoral 13 estaciones (Fig. 2).

Para cada uno de los 6 ámbitos se ha obtenido una serie representativa, resultado de promediar los datos de precipitación diarios de todas las series adscritas a cada ámbito. Las ventajas de ello, aparte de tener una mejor representatividad del conjunto de cada ámbito geográfico-hidrológico, es la minimización del peso de cualquier posible no homogeneidad no detectada en una serie particular. 


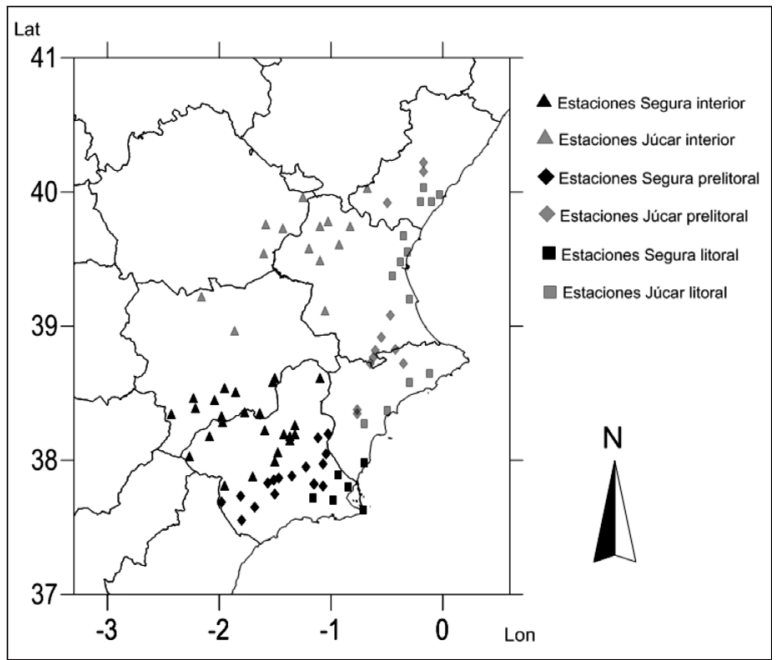

FIGURA 2. Localización del total de estaciones utilizadas para cada ámbito geográfico.

Finalmente, las series de precipitación resultantes representativas de cada conjunto territorial, se cruzan con el catálogo diario de inputs, dando como resultado la desagregación de los volúmenes de precipitación correspondientes a cada input. Estos volúmenes se agrupan en totales anuales de precipitación para cada input y zona, sobre los que posteriormente se lleva a cabo el análisis de tendencia.

\subsection{Métodos utilizados para analizar las tendencias}

El análisis de tendencia se aplicará sobre la base de los volúmenes anuales de precipitación entre 1958-2008, tanto para los totales anuales como para la desagregación en inputs.

Tradicionalmente, a la hora de valorar la pendiente de tendencia en series temporales, se ha hecho uso, y a veces abusado, de la regresión lineal simple. Aunque es un método fácil de aplicar y muchas veces útil, no es un método confiable de extracción de tendencia cuando las series temporales no son lo suficientemente largas y son ruidosas. En el caso de valores anuales de precipitación sin tendencias acusadas, sería recomendable tener al menos 100 años para extraer una tendencia confiable por este método. En nuestro caso, la ausencia de series de esa magnitud temporal nos obliga a buscar métodos de extracción de tendencia y validación mejores, para tener resultados confiables. Vamos a utilizar, por un lado, el test de Mann-Kendall para estimar la tendencia y su grado de significación, junto con la estimación Sen de pendiente. Y por otro utilizaremos una técnica relativamente más novedosa y potente, denominada Singular Spectrum Analysis (SSA), capaz de separar, en componentes principales, la tendencia del ruido, e incluso de los ciclos «ocultos» en la serie.

\subsubsection{El test de Mann-Kendall y estimación Sen}

En primer lugar se ha utilizado el test no paramétrico de Mann-Kendall (Mann, 1945; Kendall, 1975) en su versión monotónica anual, para la detección de tendencia con significancia estadística. Este test es ya ampliamente utilizado en múltiples campos de las 
ciencias naturales por su poder de extracción de tendencias confiables sin necesidad de un alto número de muestras, y tolerancia al ruido en la serie. Cuando el número de muestras a tratar es elevado $(n>40)$, como es el caso de este trabajo, se utiliza el test estadístico Z para la detección de tendencias. Además, se ha complementado con la estimación 'Sen' de pendiente de la tendencia, que complementa al test de Mann-Kendall (SEN, 1968). Para estos cálculos se ha utilizado la macro 'Makesens', desarrollada por Tino Salmi del Finnish Meteorological Institute (Salmi, T. et al, 2002).

Es importante la valoración de los grados de significación estadística de los resultados, ya que cuanto más alto es el grado de significación, la tendencia será en el mismo sentido más fiable o confiable. En este sentido, los grados de confiabilidad son: 1) Excelente: *** 99,9\% de significación; 2) Muy Buena: ** 99\% de significación; 3) Buena: * 95\% de significación; 4) Aceptable: + 90\% de significación; 4) Dudosa: (en blanco) menos del $90 \%$ de significación.

Junto con ello, también se valora la confiabilidad de la pendiente Sen (Q) mediante intervalos de confianza. Se incluyen dos, siendo el más exigente del 99\% (Qmin99 y Qmax99) y el segundo del 95\% (Qmin95 y Qmax95). Si los intervalos de confianza son laxos y entran tanto en terreno negativo como positivo, entonces la tendencia será dudosa, y en el caso contrario, será confiable.

\subsubsection{El análisis de espectro singular}

Este procedimiento, denominado SSA (Singular Spectrum Analysis), es una potente y novedosa herramienta de análisis de series temporales de reciente aceptación en el campo de las ciencias naturales y sociales. Aunque su gestación se produce hace ya algunos años, concretamente para mejorar el análisis de series paleoclimáticas (Vautard y Ghil, 1989), no se desarrolla y difunde hasta mediados de los años 90 (Elsner y Tsionis, 1996). Su uso en climatología se va difundiendo en los últimos años, con múltiples aplicaciones, como el análisis de tendencias, la detección de comportamientos cíclicos, relación de estos con índices oscilatorios, detección de rupturas o puntos de cambio, e incluso la extrapolaciónprevisión, y en múltiples variables, entre ellas temperatura y precipitación. Destacan los trabajos en esta línea en el ámbito español de Rodríguez-Puebla et al., 1998; Esteban-Parra et al, 1998 y 1999; y García el al. 2002.

Su utilidad radica en su capacidad para extraer información de la serie, consiguiendo separar en varios campos el componente tendencia, los componentes oscilatorios, y el ruido aleatorio. Descompone la serie en patrones elementales aditivos, usando filtros adaptados a los datos. Existen con anterioridad otras técnicas de descomposición en patrones aditivos, como las basadas en Fourier; pero estas parten de la extracción de componentes periódicos armónicos, construidos a partir de fases y amplitudes. La ventaja de la SSA es su capacidad de extracción de componentes quasi-periódicos, anarmónicos o irregulares (que es precisamente como suelen darse las oscilaciones en la naturaleza), ya que la base de su análisis no son las fases y amplitudes, sino la varianza, concretamente la matriz de covarianzas. Ello pues mejora sustancialmente la extracción de información oculta en la serie temporal. $\mathrm{Su}$ valor ha sido precisamente demostrado en series cortas y ruidosas.

En este trabajo se utiliza únicamente la capacidad de la SSA para extraer la tendencia. El componente 1, el de mayor peso, se asume que es la tendencia. Cuando su peso es muy superior al segundo y resto de componentes, explicando por sí solo la mayor parte del porcentaje de la serie, como es el caso en este trabajo (más del 70-80\% generalmente), predomina en la serie la tendencia sobre cualquier componente oscilatorio, y los restantes componentes conforman en su mayor parte el ruido, que proporciona el grado de aleatoriedad 
interanual de la serie. Los cálculos se han realizado a través del método 'Caterpillar-SSA', utilizando el paquete de software del mismo nombre (Alexandrov, 2005 y 2006), (http:// www.gistatgroup.com/cat/), (http://www.pdmi.ras.ru/ theo/autossa/english/index.htm)

\section{Resultados}

\subsection{Análisis de las tendencias}

Tabla 1

RESULTADOS DEL TEST DE MANN-KENDALL Y DE LA ESTIMACIÓN SEN DE PENDIENTE. ELABORACIÓN PROPIA

\begin{tabular}{|c|c|c|c|c|c|c|c|c|c|c|}
\hline \multirow[b]{2}{*}{ Series } & \multirow[b]{2}{*}{ Comienza } & \multirow[b]{2}{*}{ Acaba } & \multirow[b]{2}{*}{$\mathrm{n}$} & \multicolumn{2}{|c|}{ Mann-Kendall trend } & \multicolumn{5}{|c|}{ Sen's slope estimate } \\
\hline & & & & Test Z & Signific. & Q & Qmin99 & Qmax99 & Qmin95 & Qmax95 \\
\hline Segura interior, total & 1958 & 2008 & 51 & 0.24367 & & 0.35 & -2.713 & 2.8127 & -1.918 & 2.27021 \\
\hline Júcar interior, total & 1958 & 2008 & 51 & -1.3158 & & -1.439 & -4.441 & 1.5117 & -3.701 & 0.74489 \\
\hline Segura prelitoral, total & 1958 & 2008 & 51 & -0.19493 & & -0.228 & -2.563 & 2.2827 & -1.871 & 1.71942 \\
\hline Júcar prelitoral, total & 1958 & 2008 & 51 & 0.50358 & & 0.685 & -3.495 & 5.6169 & -2.468 & 4.59883 \\
\hline Segura litoral, total & 1958 & 2008 & 51 & 0.50358 & & 0.423 & -1.972 & 2.6858 & -1.474 & 2.16835 \\
\hline Júcar litoral, total & 1958 & 2008 & 51 & 0.30864 & & 0.32 & -3.179 & 3.6799 & -2.375 & 2.78214 \\
\hline Segura interior, atlántica & 1958 & 2008 & 51 & -1.61637 & & -0.64 & -1.62 & 0.3715 & -1.41 & 0.09779 \\
\hline Júcar interior, atlántica & 1958 & 2008 & 51 & -2.98095 & $* *$ & -1.514 & -2.474 & -0.2702 & -2.24 & -0.5524 \\
\hline Segura prelitoral, atlántica & 1958 & 2008 & 51 & -0.71475 & & -0.212 & -0.966 & 0.4958 & -0.784 & 0.32994 \\
\hline Júcar prelitoral, atlántica & 1958 & 2008 & 51 & -1.08838 & & -0.468 & -1.519 & 0.6912 & -1.326 & 0.43665 \\
\hline 1, atlántica & 1958 & 2008 & 51 & -0.89344 & & -0.183 & -0.851 & 0.4248 & -0.689 & 0.27847 \\
\hline Júcar litoral, atlántica & 1958 & 2008 & 51 & -0.77973 & & -0.363 & -1.487 & 0.7894 & -1.239 & 0.53507 \\
\hline Segura i & 1958 & 2008 & 51 & 0.86095 & & 0.508 & -1.25 & 2.4196 & -0.924 & 1.93141 \\
\hline Jucar interior, retroceso & 1958 & 2008 & 51 & 0.76349 & & 0.325 & -1.131 & 1.7377 & -0.807 & 1.33238 \\
\hline prelitoral, retr & 88 & 2008 & 51 & 0.12996 & & 0.05 & -1.652 & 2.0491 & -1.279 & 1.48491 \\
\hline Jucar prelitoral, retroceso & 1958 & 2008 & 51 & 1.03964 & & 1.232 & -2.238 & 4.7269 & -1.202 & 4.12814 \\
\hline Segura litoral, retroceso & 1958 & 2008 & 51 & 0.35738 & & 0.37 & -1.844 & 2.5596 & -1.287 & 1.86343 \\
\hline Jucar litoral, retroceso & 1958 & 2008 & 51 & 0.92593 & & 0.84 & -1.447 & 3.0326 & -0.828 & 2.42145 \\
\hline Segura interior, retroceso seca & 1958 & 2008 & 51 & -1.44575 & & -0.147 & -0.446 & 0.1187 & -0.376 & 0.05715 \\
\hline Jucar interior, retroceso seca & 1958 & 2008 & 51 & -3.02146 & $* *$ & -0.397 & -0.773 & -0.0614 & -0.69 & -0.1474 \\
\hline Segura prelitoral, retroceso seca & 1958 & 2008 & 51 & -1.33204 & & -0.164 & -0.479 & 0.1407 & -0.403 & 0.06822 \\
\hline Jucar & 1958 & 2008 & 51 & -2.13621 & $*$ & -0.522 & -1.249 & 0.1292 & -1.103 & -0.0406 \\
\hline Segura litoral, retroceso seca & 1958 & 2008 & 51 & -0.66602 & & -0.088 & -0.419 & 0.2364 & -0.336 & 0.16344 \\
\hline Jucar li & 1958 & 2008 & 51 & -2.66409 & $* *$ & -0.537 & -1.089 & -0.0161 & -0.933 & -0.1586 \\
\hline Segura interior, convectiva & 1958 & 2008 & 51 & 0.82847 & & 0.246 & -0.63 & 1.0562 & -0.377 & 0.82307 \\
\hline Jucar interior, convectiva & 1958 & 2008 & 51 & -0.69851 & & -0.344 & -1.475 & 0.7397 & -1.183 & 0.46351 \\
\hline Segura prelitoral, convectiva & 1958 & 2008 & 51 & 0.22742 & & 0.033 & -0.642 & 0.7254 & -0.472 & 0.58328 \\
\hline Jucar prelitoral, convectiva & 1958 & 2008 & 51 & 0.76349 & & 0.364 & -0.754 & 1.4389 & -0.453 & 1.11757 \\
\hline Segura litoral, convectiva & 1958 & 2008 & 51 & 0.53607 & & 0.144 & -0.442 & 0.7348 & -0.375 & 0.54265 \\
\hline Jucar litoral, convectiva & 1958 & 2008 & 51 & 0.32489 & & 0.106 & -1.133 & 1.1254 & -0.77 & 0.90017 \\
\hline
\end{tabular}




\subsubsection{Sectores interiores}

Con relación a los totales anuales para cada uno de los sectores se aprecian diferencias. Así, mientras en el sector interior del Segura no se aprecia ninguna tendencia en la precipitación (Fig. 3), ésta si se observa en el sector interior del Júcar. De hecho, el test de Mann-Kendall arroja una tendencia al descenso, si bien el grado de significación no es suficientemente elevado para ser confiable. Igualmente la pendiente Sen es negativa, pero los intervalos de confianza ponen en duda la tendencia (Tabla 1). Sin embargo la tendencia extraída por SSA sí muestra un claro descenso, al menos hasta mediados de los 90 (Fig. 4).

Sin embargo, en el análisis desagregado por inputs sí aparecen tendencias al descenso de las precipitaciones confiables, básicamente en la componente atlántica y la de origen septentrional «retroceso seca». Se hace así notar como, para el caso del sector 'Júcar interior', tanto las precipitaciones por advecciones atlánticas como por 'retroceso seca', arrojan en el test de Mann-Kendall valores de tendencia negativa próximos a -3, y en este caso la significación es muy buena (superior al 99\%) en ambos casos (Tabla 1). Del mismo modo, la estimación Sen y sus intervalos de confianza en ambos casos no ofrecen duda de la tendencia negativa.

Si se compara este sector Júcar interior con el Segura interior, aunque las tendencias observadas en el segundo caso no alcanzan a tener suficientes niveles de confiabilidad en el caso del test de Mann-Kendall y la estimación Sen (Tabla 1), se observa un comportamiento paralelo con el primero. De hecho, al observar la tendencia extraída mediante SSA, se aprecia un paralelismo muy claro en el descenso de las 'atlánticas' y las de 'retroceso seca', que también se ve en el caso del sector 'Segura interior' (Figs. 5 y 9), como en el del 'Jucar interior' (Figs. 6 y 10). En ambos casos el descenso de las precipitaciones atlánticas ronda el $20 \%$, aunque en el caso de las de 'retroceso seca' (con un peso relativo muy bajo), su disminución es muy acusada.

Para las advecciones mediterráneas ('retroceso'), los resultados muestran igualmente un paralelismo entre ambos casos, aunque sin detectarse ninguna tendencia negativa, sino neutra, o incluso ligeramente positiva. Para los dos ámbitos, en el test de Mann-Kendall y en la estimación Sen se muestra una ligera tendencia positiva, pero sin buena confiabilidad (Tabla 1). La misma conclusión se puede extraer al observar el componente de tendencia extraído mediante SSA (Figs. 7 y 8).

En el caso de las precipitaciones de origen convectivo el comportamiento sí es un tanto diferente entre el observado en el sector interior del Júcar y en el del Segura, aunque en este caso ninguna tendencia presenta suficientes niveles de confianza en el test de MannKendall y estimación Sen (Tabla 1). La tendencia en el sector del Segura es más dudosa, porque los resultados obtenidos del análisis SSA muestran una tendencia plana (Fig. 11). Sin embargo en el sector del Júcar, el análisis por SSA sí que apoya, aunque con reservas, una tendencia negativa (Fig. 12).

En resumen, para el sector interior, lo más significativo es la tendencia al descenso de las precipitaciones en los inputs 'atlántica' y 'retroceso seca'. Para el sector Júcar interior este descenso es más significativo en cuanto que el peso de las precipitaciones atlánticas es en esta área porcentualmente mayor, lo que también llega a traducirse en una influencia negativa sobre los totales anuales. En el sector 'Segura interior' esta tendencia parece verse más compensada en los totales anuales básicamente por las advecciones mediterráneas ('retroceso'). 


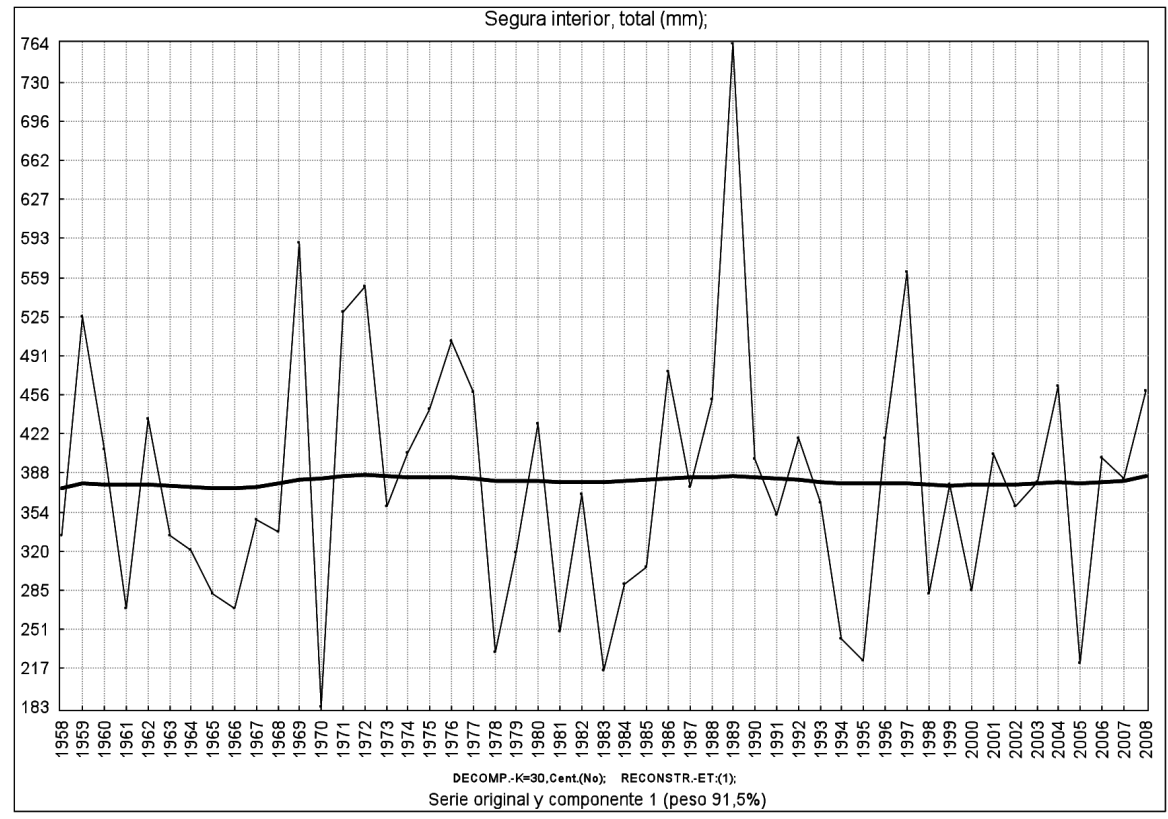

FIGURA 3. Precipitación total para el sector 'Segura interior' y tendencia extraída mediante SSA.

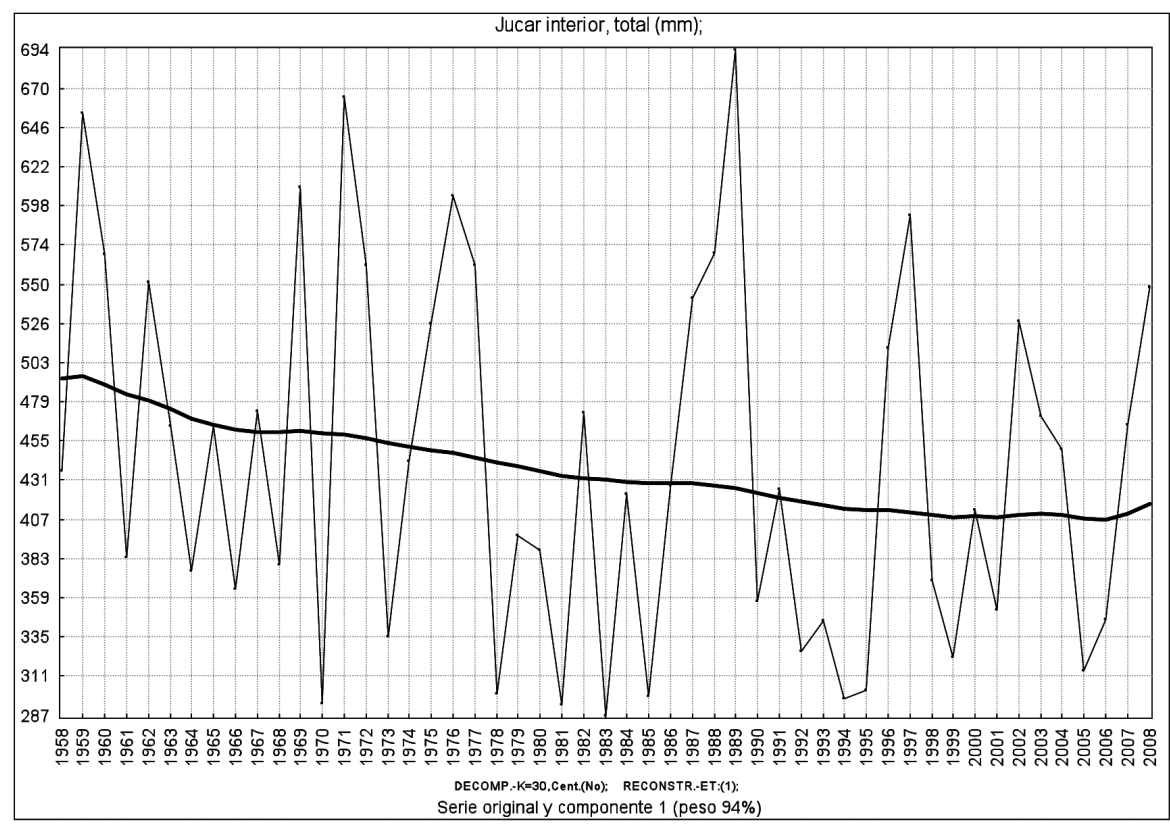

FIGURA 4. Precipitación total para el sector 'Júcar interior' y tendencia extraída mediante SSA. 


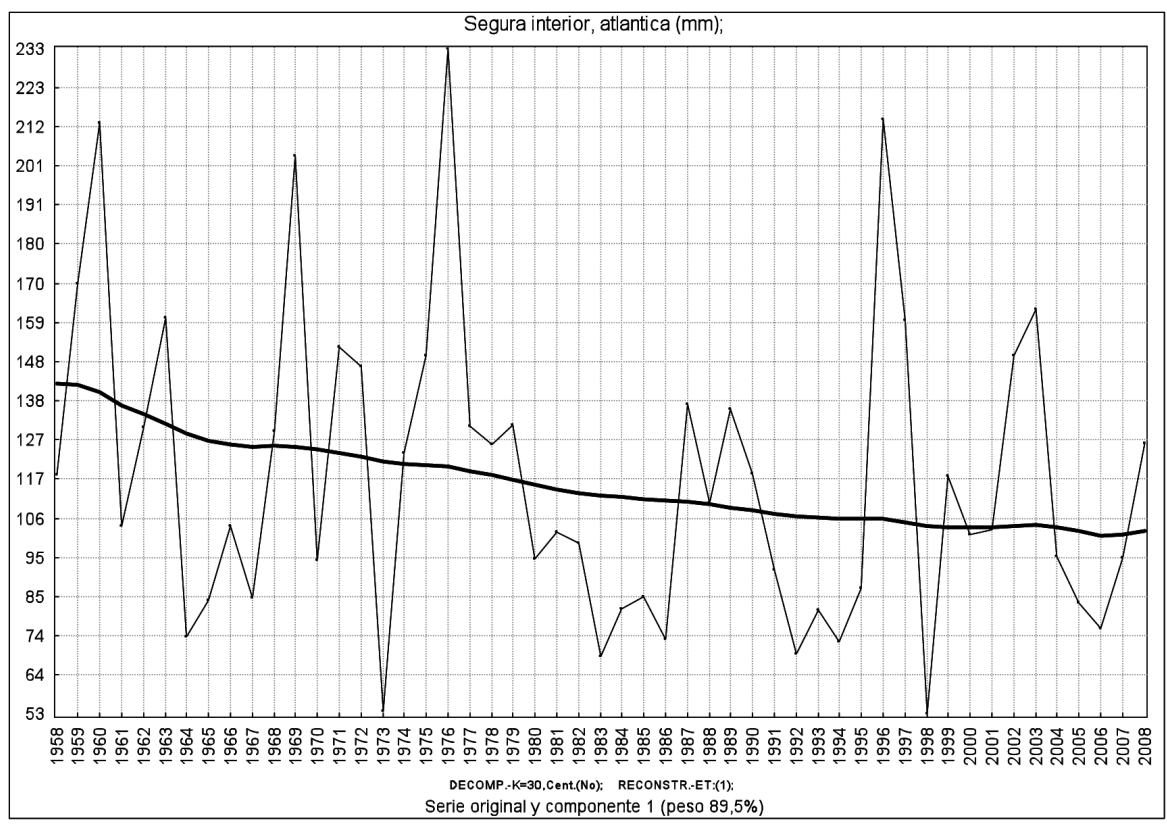

FIGURA 5. Precipitación por advección atlántica para el sector 'Segura interior' y tendencia extraída mediante SSA.

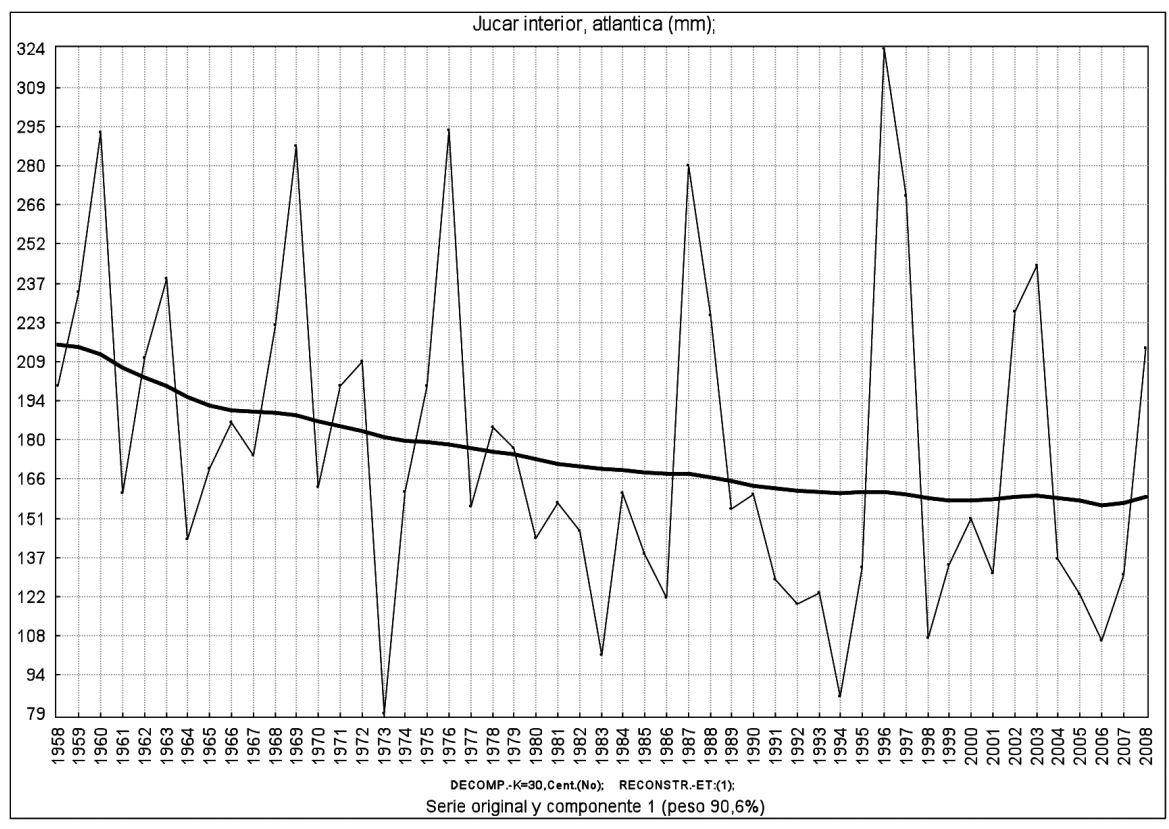

FIgURA 6. Precipitación por advección atlántica para el sector 'Júcar interior' y tendencia extraída mediante SSA. 


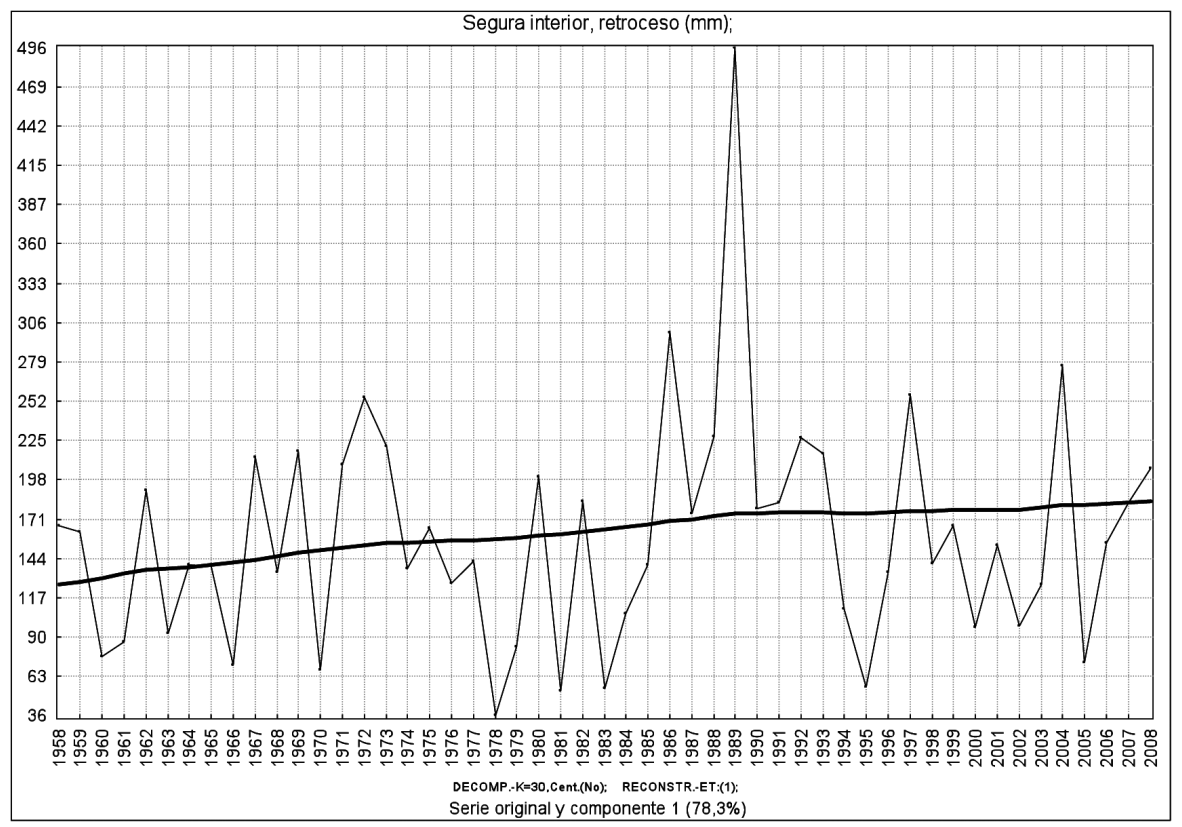

FiguRA 7. Precipitación por advección mediterránea, o de retroceso, para el sector ‘Segura interior' y tendencia extraída mediante SSA.

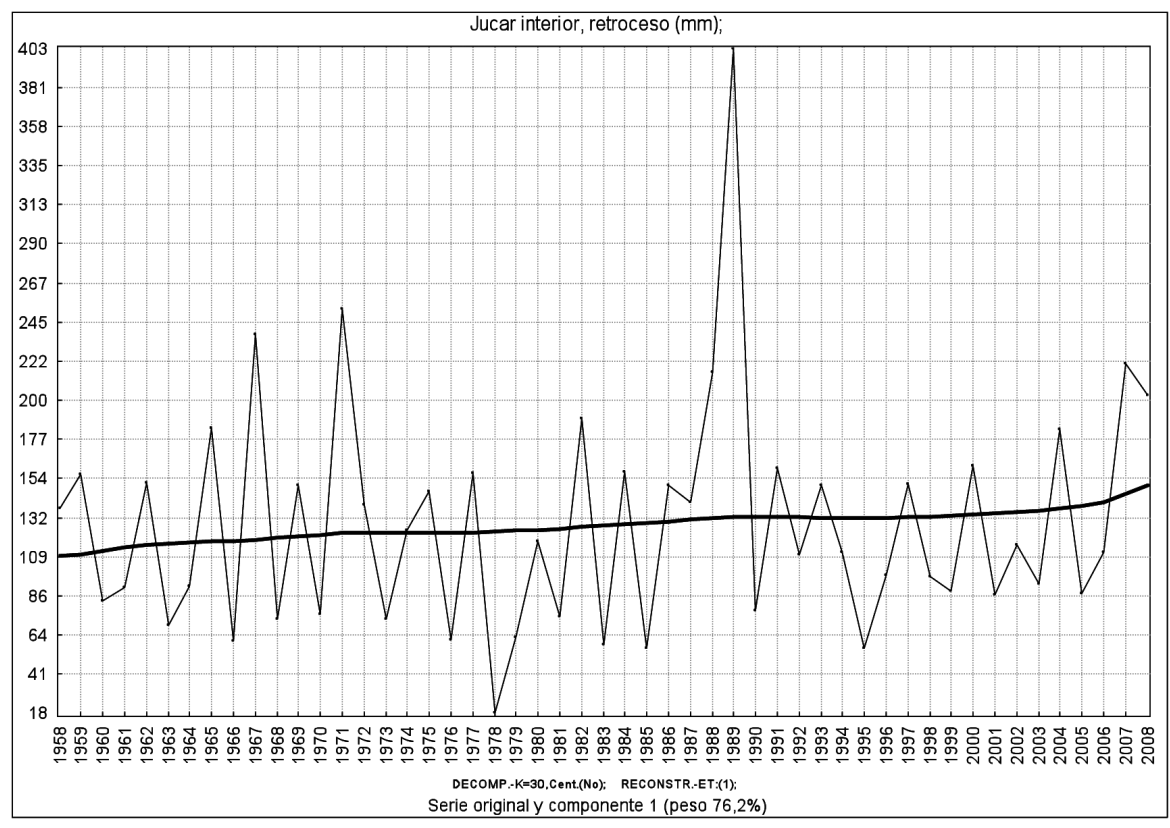

FIGURA 8. Precipitación por advección mediterránea, o de retroceso, para el sector 'Júcar interior' y tendencia extraída mediante SSA. 


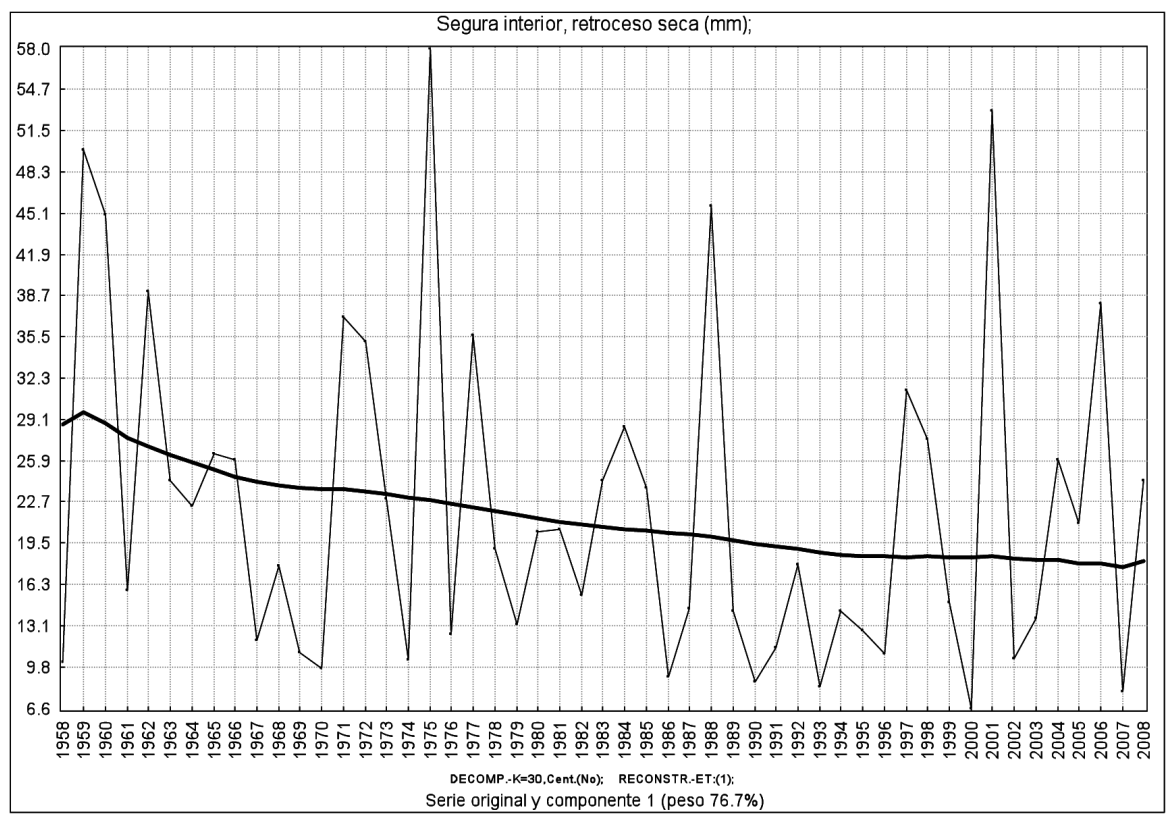

FIgURA 9. Precipitación por advección continental, o de retroceso seca, para el sector 'Segura interior' y tendencia extraída mediante SSA.

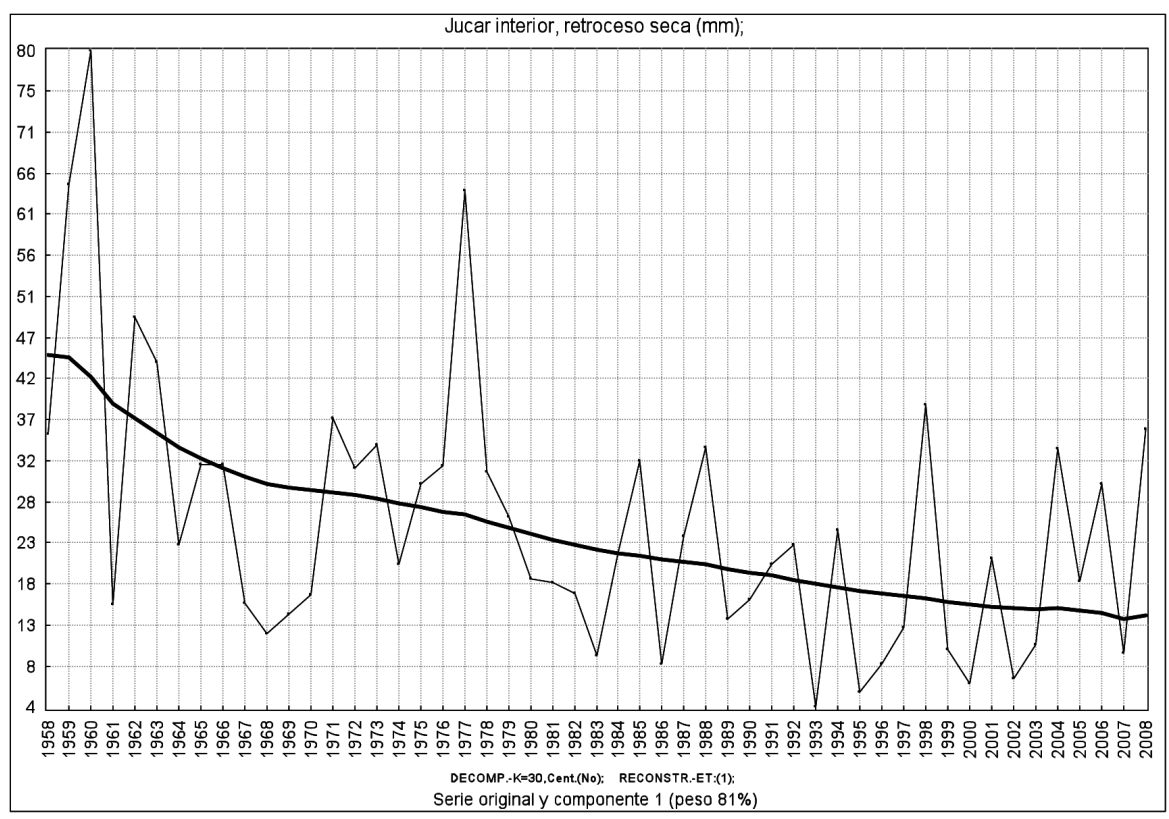

FIgURA 10. Precipitación por advección continental, o de retroceso seca, para el sector 'Júcar interior' y tendencia extraída mediante SSA. 


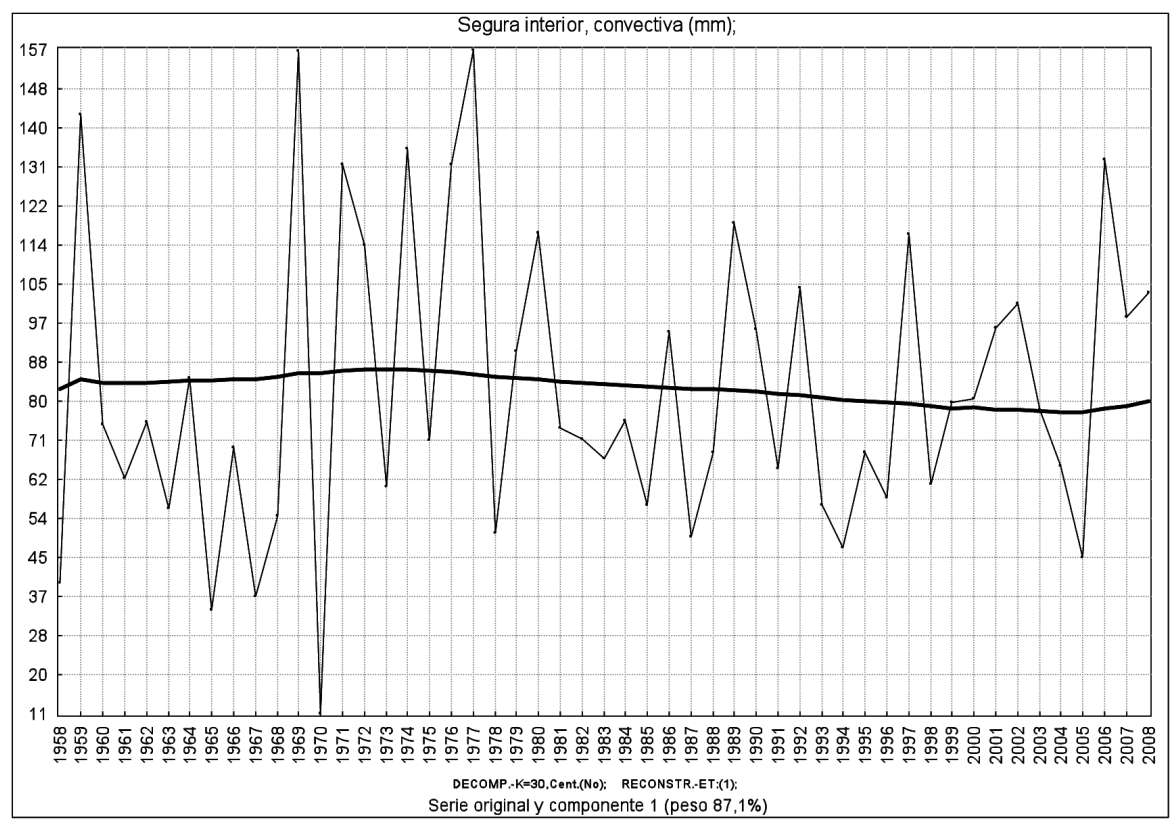

FIGURA 11. Precipitación por situaciones convectivas para el sector 'Segura interior' y tendencia extraída mediante SSA.

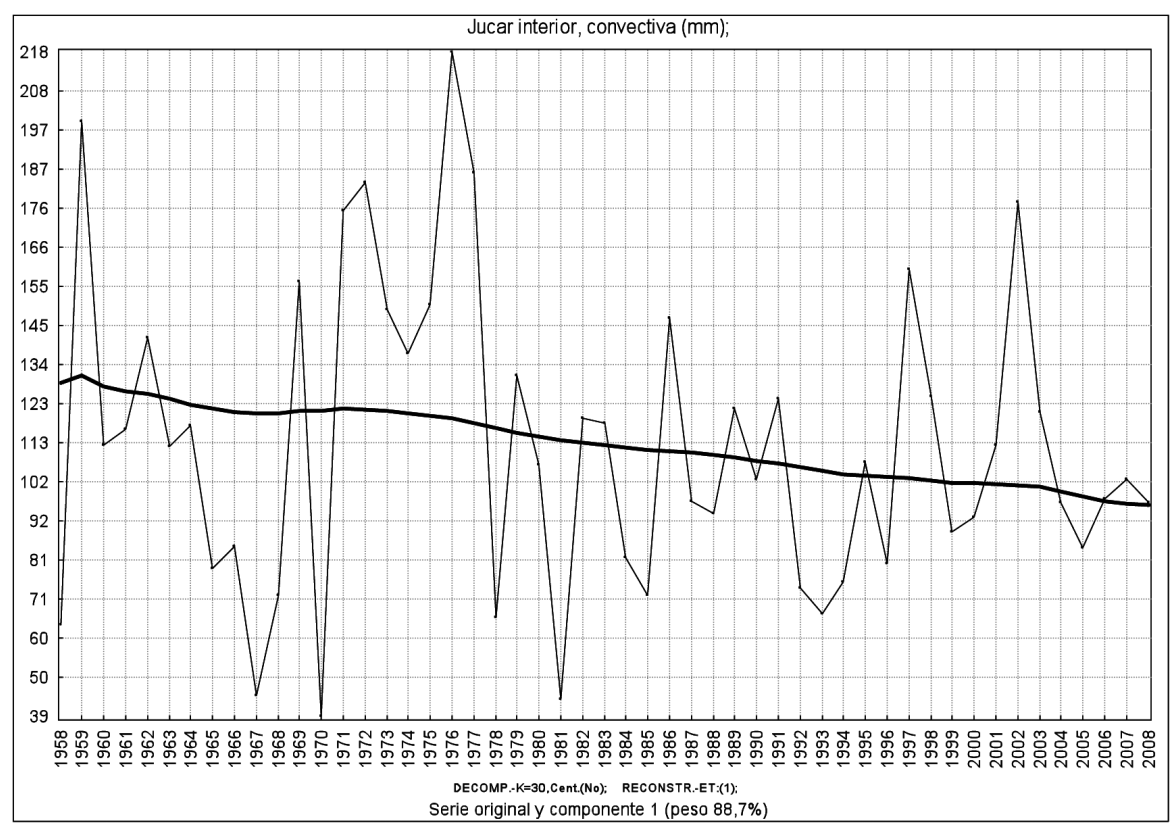

FIGURA 12. Precipitación por situaciones convectivas para el sector 'Júcar interior' y tendencia extraída mediante SSA. 


\subsubsection{Sectores prelitorales}

En general, para el caso de los sectores prelitorales, encontramos tendencias en conjunto más débiles o dudosas. No obstante, sí existe un comportamiento reflejo al ya observado en los sectores interiores, en cuanto a las diferencias presentes entre los distintos inputs. Superpuesto a ello, y a diferencia de lo que ocurría en el interior, en el sector prelitoral del Júcar parece haber una tendencia subyacente al ascenso, liderada por las advecciones mediterráneas ('retroceso'), caso que ya no alcanza a producirse en el caso del prelitoral del Segura.

Los totales anuales muestran una tendencia neutra en el caso del análisis SSA para el sector «Segura prelitoral» (Fig. 13), mientras que aparece una leve tendencia al ascenso (dudosa) en el caso del sector 'Júcar prelitoral' (Fig. 14). Sin embargo, en la desagregación por inputs, para el caso de las precipitaciones de origen atlántico, esta leve pendiente de ascenso desaparece en el sector 'Júcar prelitoral' (Fig. 16), e incluso se vuelve negativa en el caso de las de 'retroceso seca' (Fig. 20). En el sector 'Segura prelitoral', no sólo se vuelve negativa para el caso de 'retroceso seca' (Fig. 19), sino también para las 'atlánticas' (Fig. 15). Las 'convectivas' en ambos casos muestran tendencias poco precisas o neutras (Figs. 21 y 22).

La estructura observada en las tendencias para el interior, se deja notar también en el sector prelitoral, aunque de forma más débil, y apreciándose el cambio de los pesos porcentuales de cada input, sobre todo a la hora de comparar los sectores interior y prelitoral del Júcar. Es pues de destacar una «reversión» de fondo que se produce entre estos últimos sectores del Júcar en la tendencia de los totales anuales, al tener mucho más peso las situaciones de 'retroceso' en el prelitoral que en el interior. Además, ahora el descenso de las precipitaciones de origen atlántico es menor y dudoso, mientras que las de 'retroceso' muestran una incipiente tendencia al ascenso, con reservas.

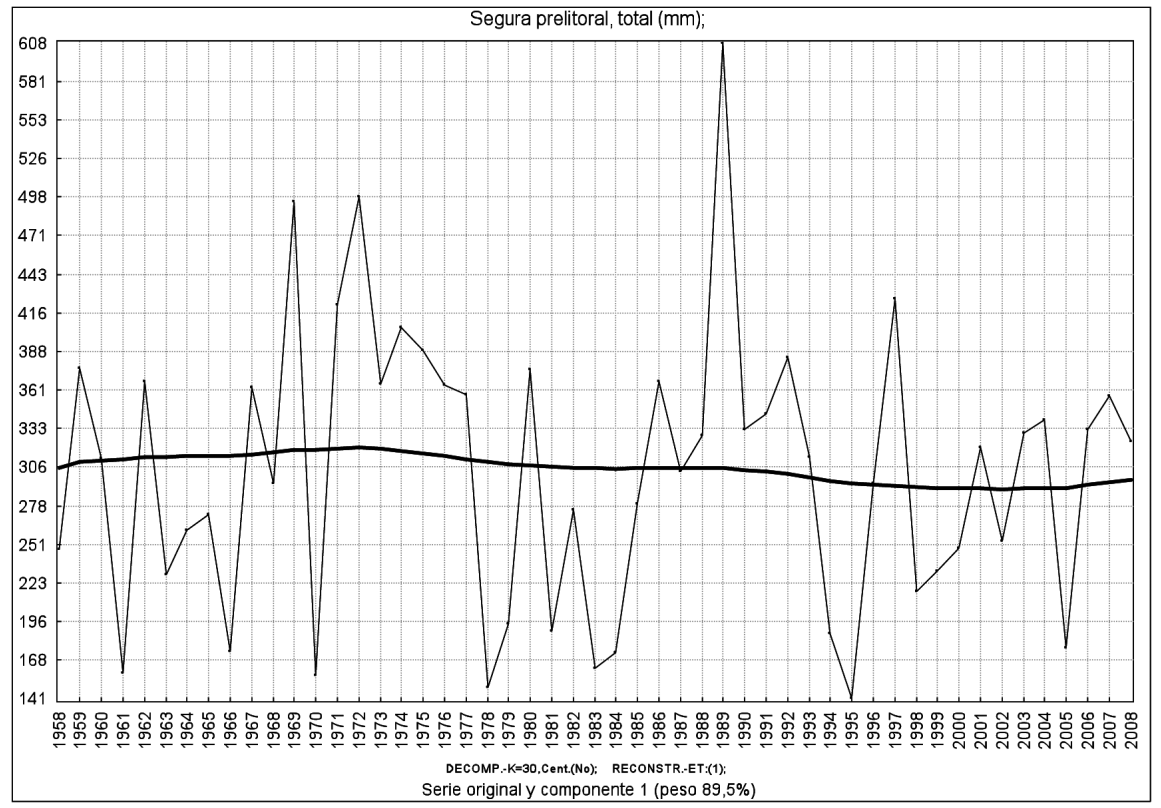

FIgURA 13. Precipitación total para el sector 'Segura prelitoral' y tendencia extraída mediante SSA. 


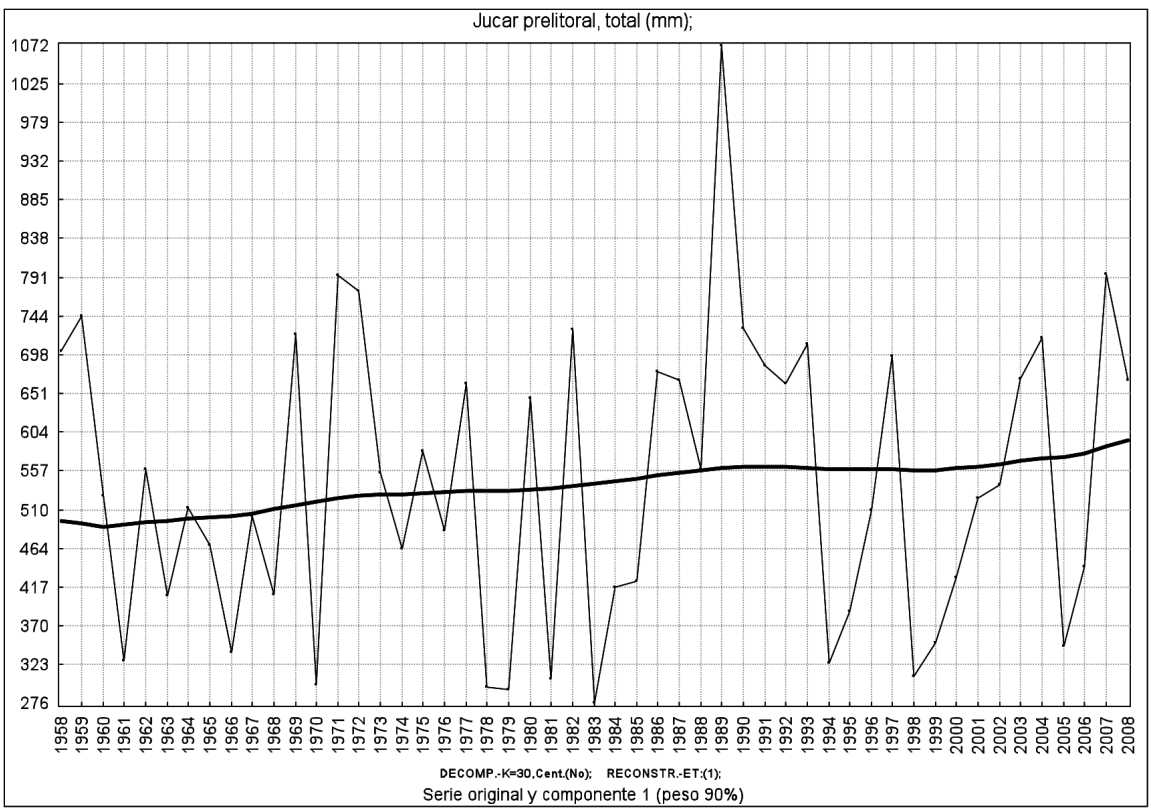

FIGURA 14. Precipitación total para el sector 'Jucar prelitoral' y tendencia extraída mediante SSA.

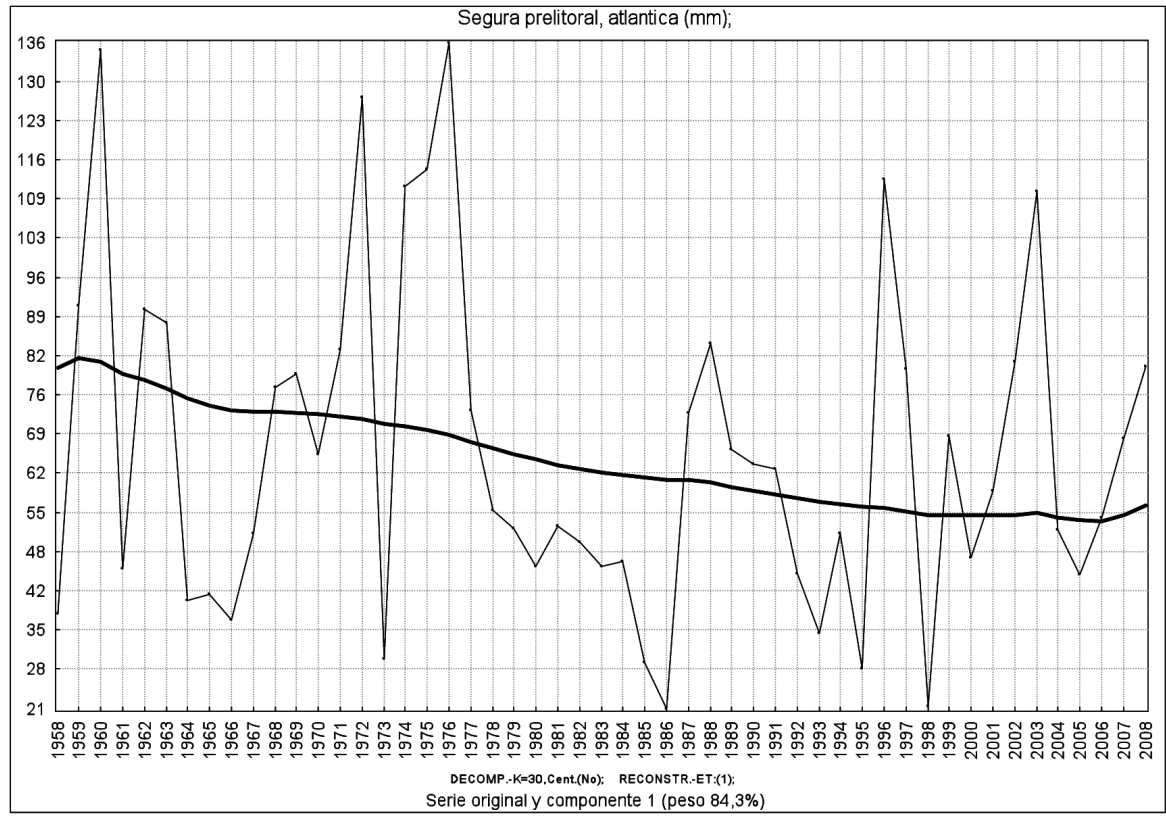

FIGURA 15. Precipitación por advección atlántica para el sector 'Segura prelitoral' y tendencia extraída mediante SSA. 


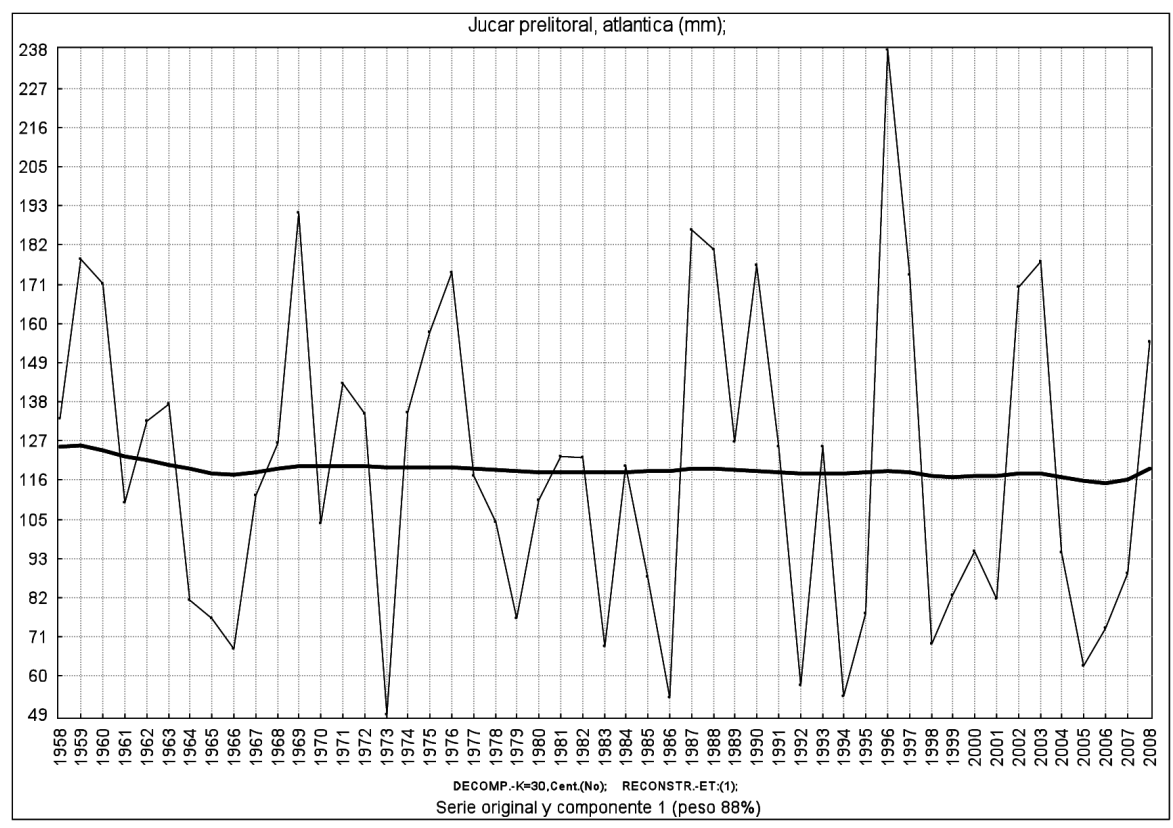

FIGURA 16. Precipitación por advección atlántica para el sector 'Júcar prelitoral' y tendencia extraída mediante SSA.

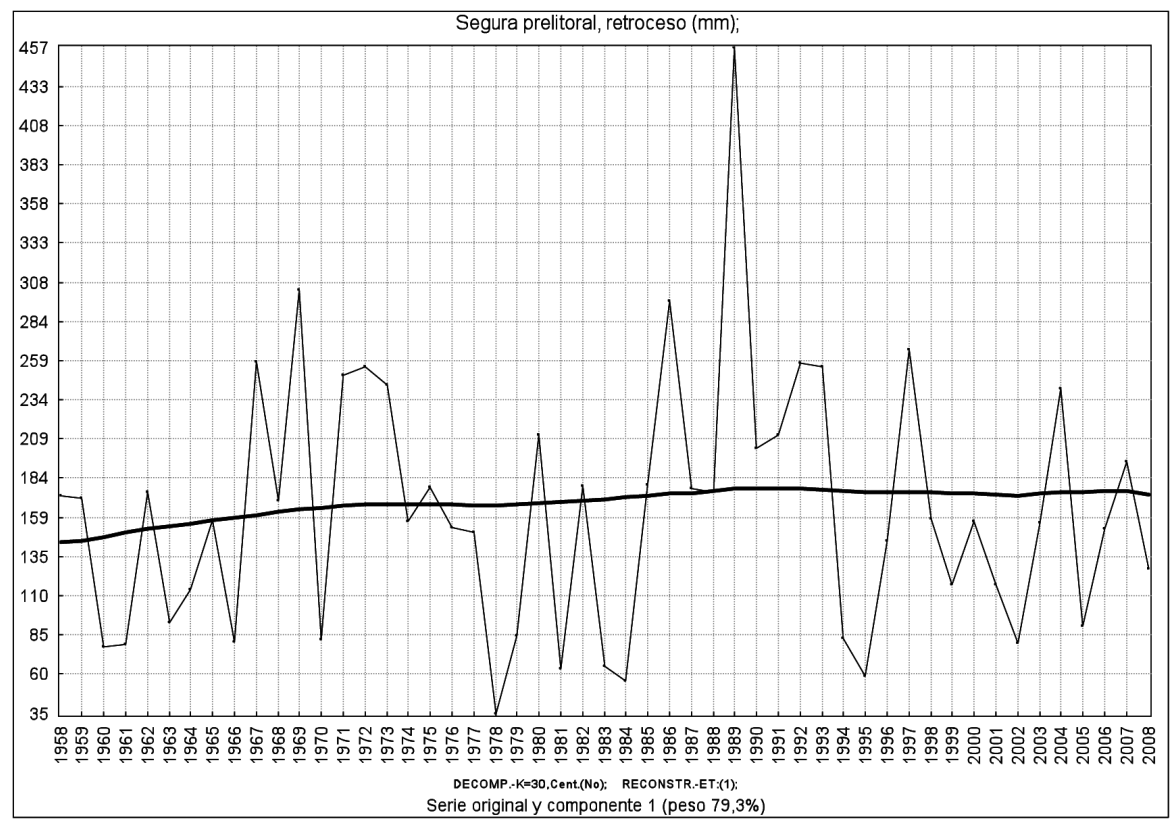

FIGURA 17. Precipitación por advección mediterránea, o de retroceso, para el sector 'Segura prelitoral' y tendencia extraída mediante SSA. 


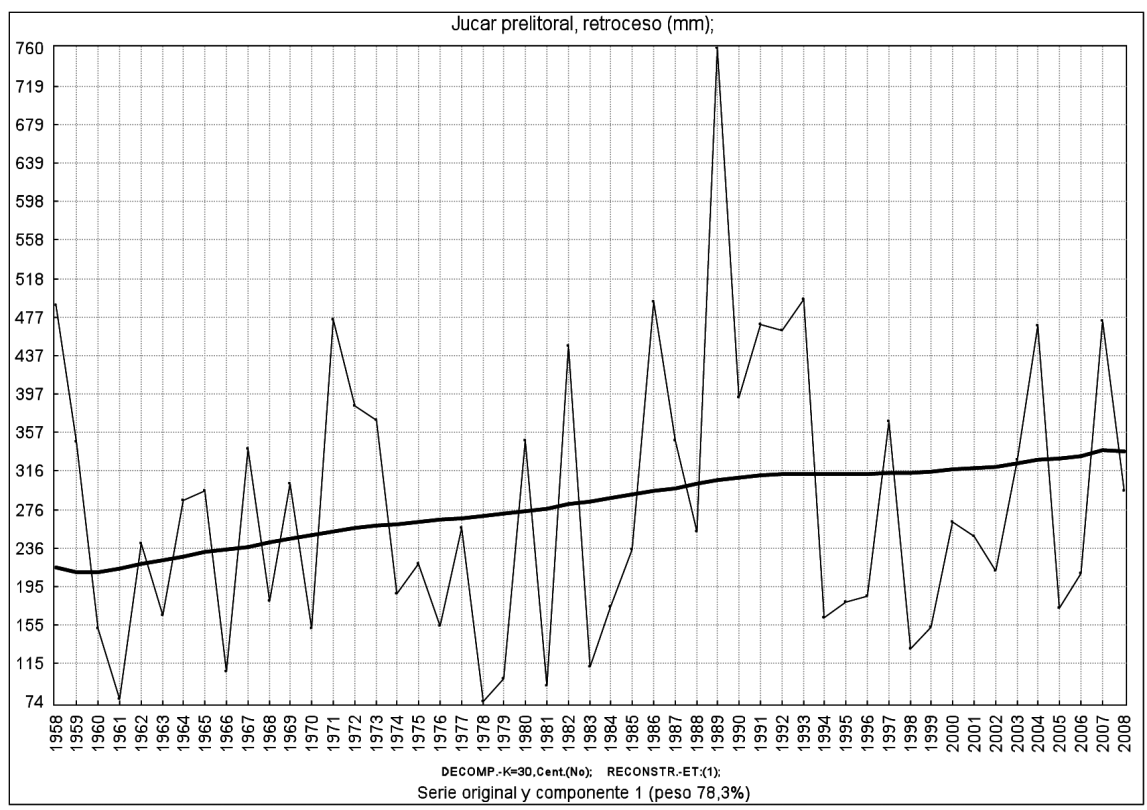

FIGURA 18. Precipitación por advección mediterránea, o de retroceso, para el sector 'Júcar prelitoral' y tendencia extraída mediante SSA.

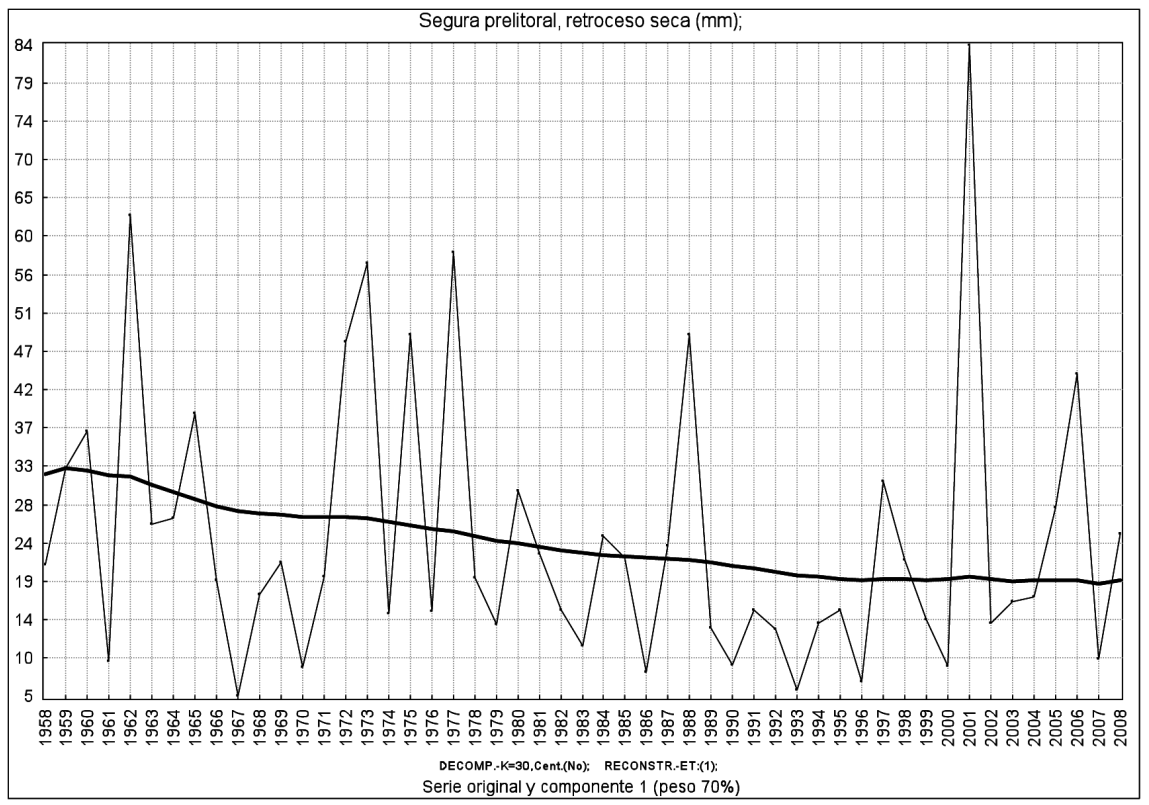

FIgURA 19. Precipitación por advección continental, o de retroceso seca, para el sector 'Segura prelitoral' y tendencia extraída mediante SSA. 


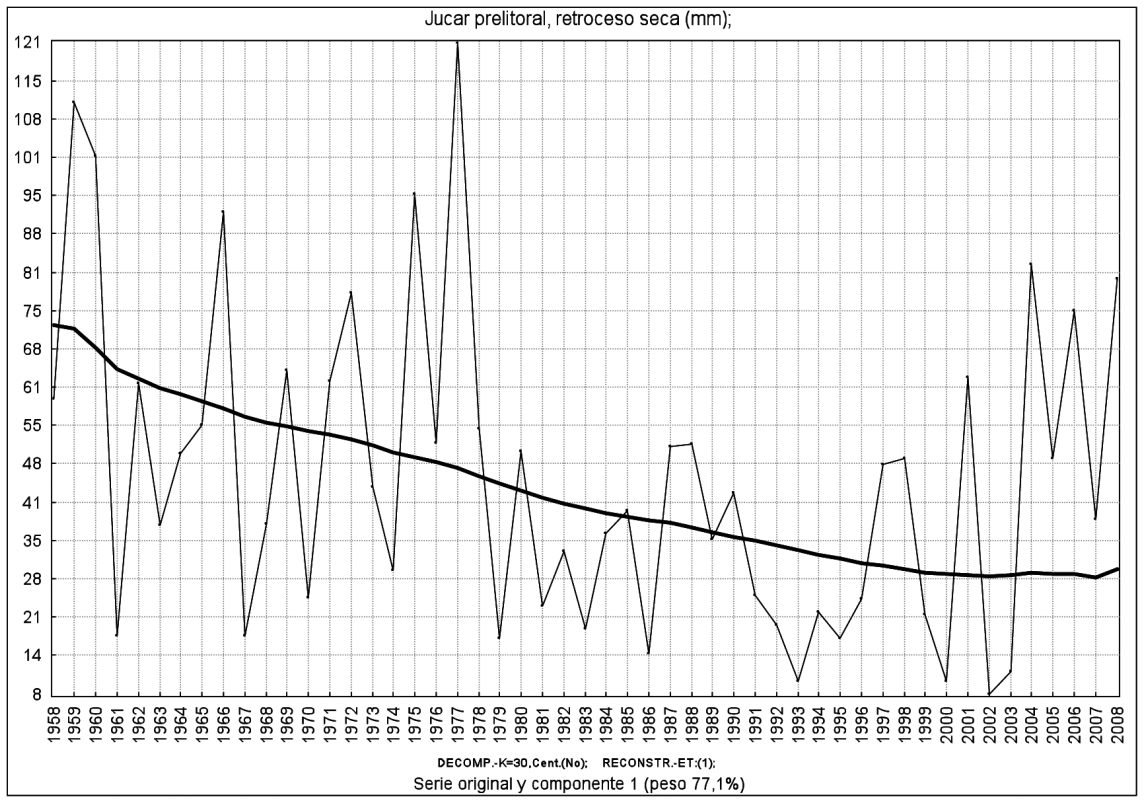

Figura 20. Precipitación por advección continental, o de retroceso seca, para el sector 'Júcar prelitoral' y tendencia extraída mediante SSA.

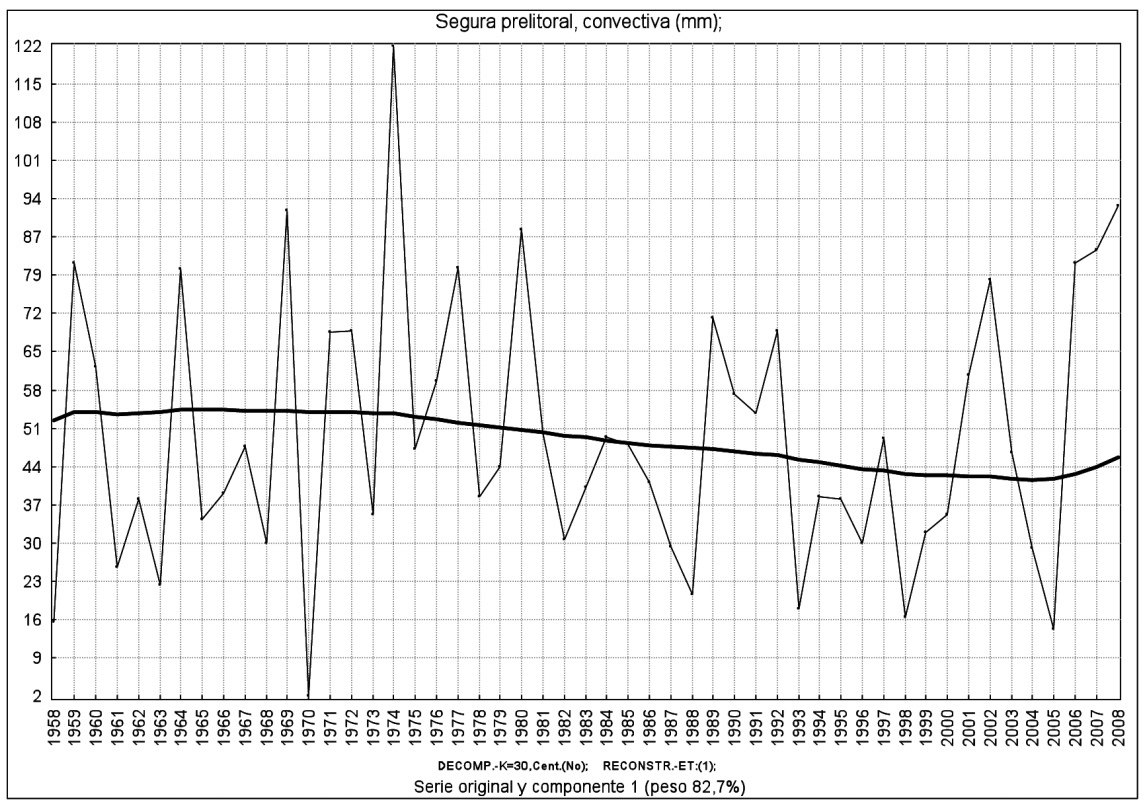

FIGURA 21. Precipitación por situaciones convectivas para el sector 'Segura prelitoral' y tendencia extraída mediante SSA. 


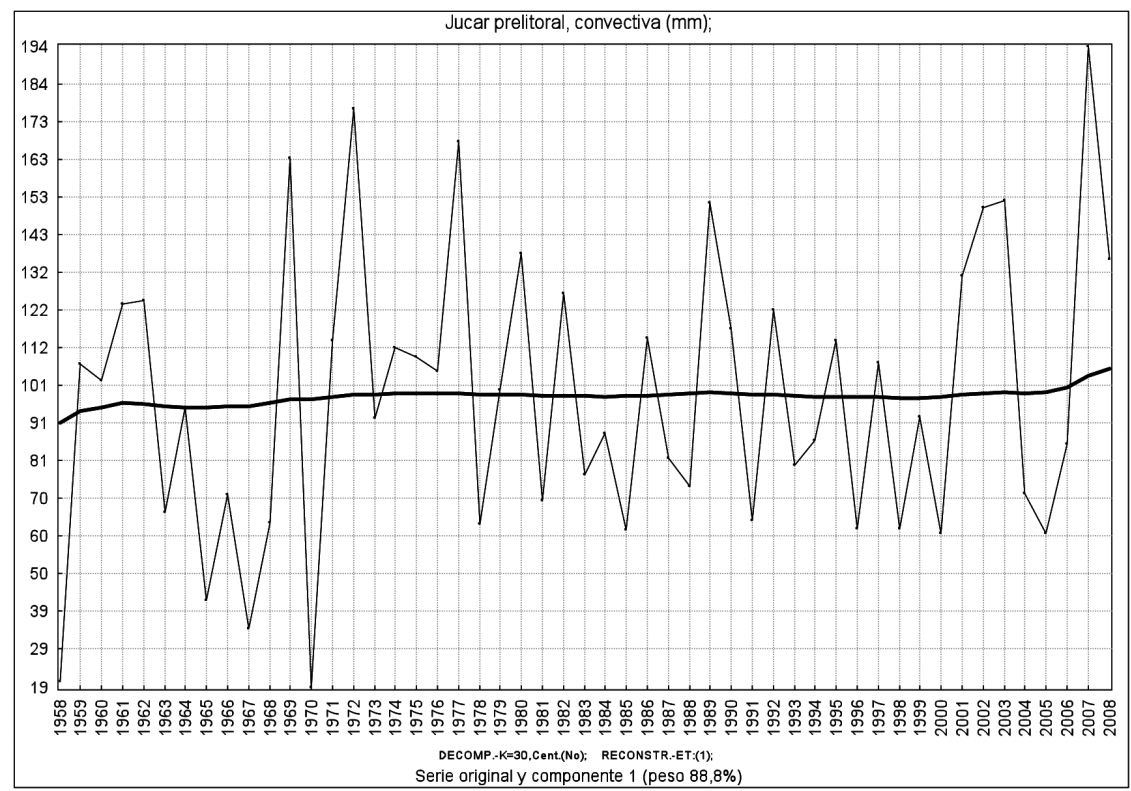

Figura 22. Precipitación por situaciones convectivas para el sector 'Júcar prelitoral' y tendencia extraída mediante SSA.

Por el contrario, en el caso del Segura se observa un mayor paralelismo entre el sector interior y el prelitoral. Se puede decir que el prelitoral del Júcar tiende a un comportamiento más próximo al del litoral, mientras que el prelitoral del Segura tiene más paralelismos con el interior. En cualquier caso, casi ninguna tendencia observada en estos sectores prelitorales alcanza a tener buenos niveles de significación y confianza en el test de Mann-Kendall y estimación Sen (Tabla 1), salvo para el caso de las situaciones de 'retroceso seca' en el sector 'Júcar prelitoral', con un resultado del test de Mann-Kendall inferior a -2, con buena significación. Esto reproduce lo ya observado en el sector 'Júcar interior', notando también que, en paralelo, para el sector 'Segura prelitoral' se observa también una pendiente negativa en este input, aunque ya no tenga el mismo grado de confianza.

\subsubsection{Sectores litorales}

Tanto el sector litoral del Segura como el del Júcar tienen un comportamiento similar en lo que a tendencias se refiere. En ambos casos los totales anuales no muestran tendencia, tanto en los resultados de los tests (Tabla 1) como en el análisis SSA (Figs. 23 y 24). Sin embargo, al realizar el análisis desagregado por inputs, sí se detectan tendencias.

Se destaca la tendencia negativa observada, para el sector 'Jucar litoral' en el input 'retroceso seca', con muy buen grado de significación en el test de Mann-Kendall (>99\%), y un valor en el mismo inferior a -2,5. Igualmente la estimación Sen da un valor negativo, y con sus intervalos de confianza al $99 \%$ y $95 \%$ en negativo (Tabla 1). Todo ello confirmado por la tendencia extraída mediante SSA con una pendiente negativa muy acusada mostrando un descenso incluso próximo al 50\% (Fig. 30). Es un hecho muy significativo, aunque el peso relativo de este input sea bajo. 


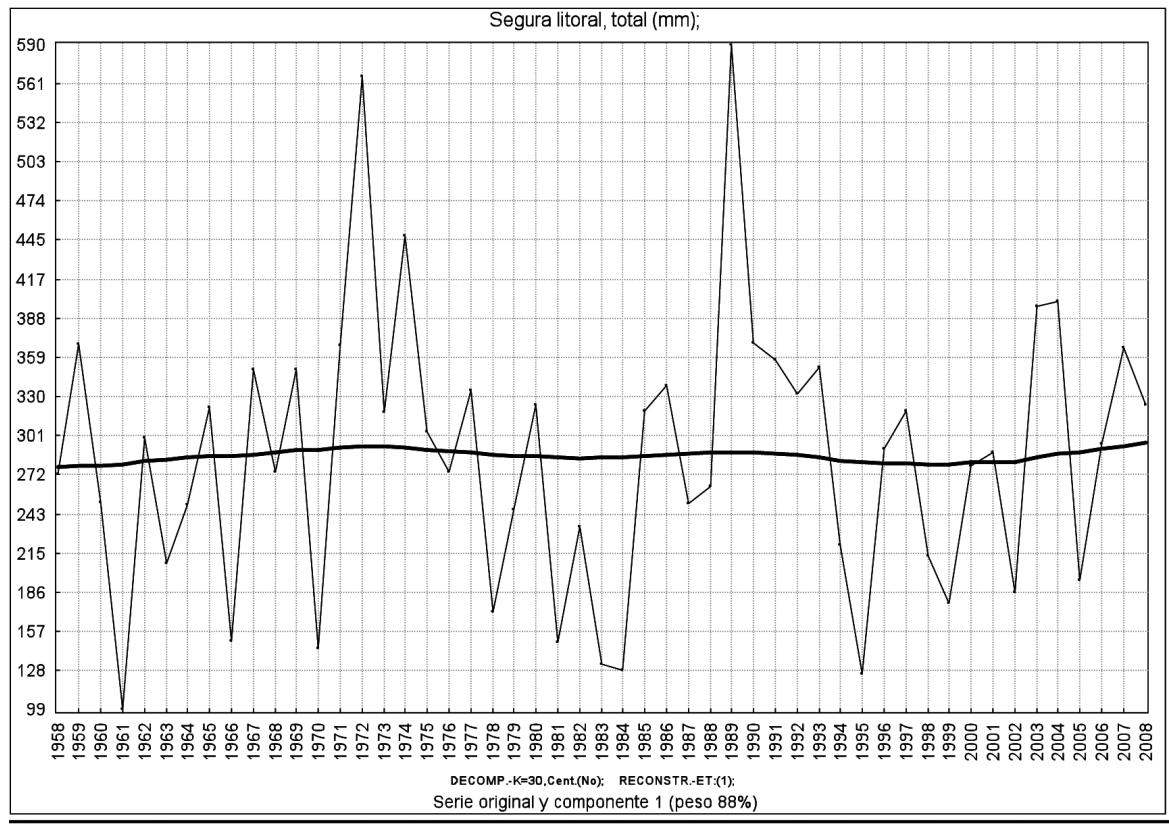

FIGURA 23. Precipitación total para el sector 'Segura litoral' y tendencia extraída mediante SSA.

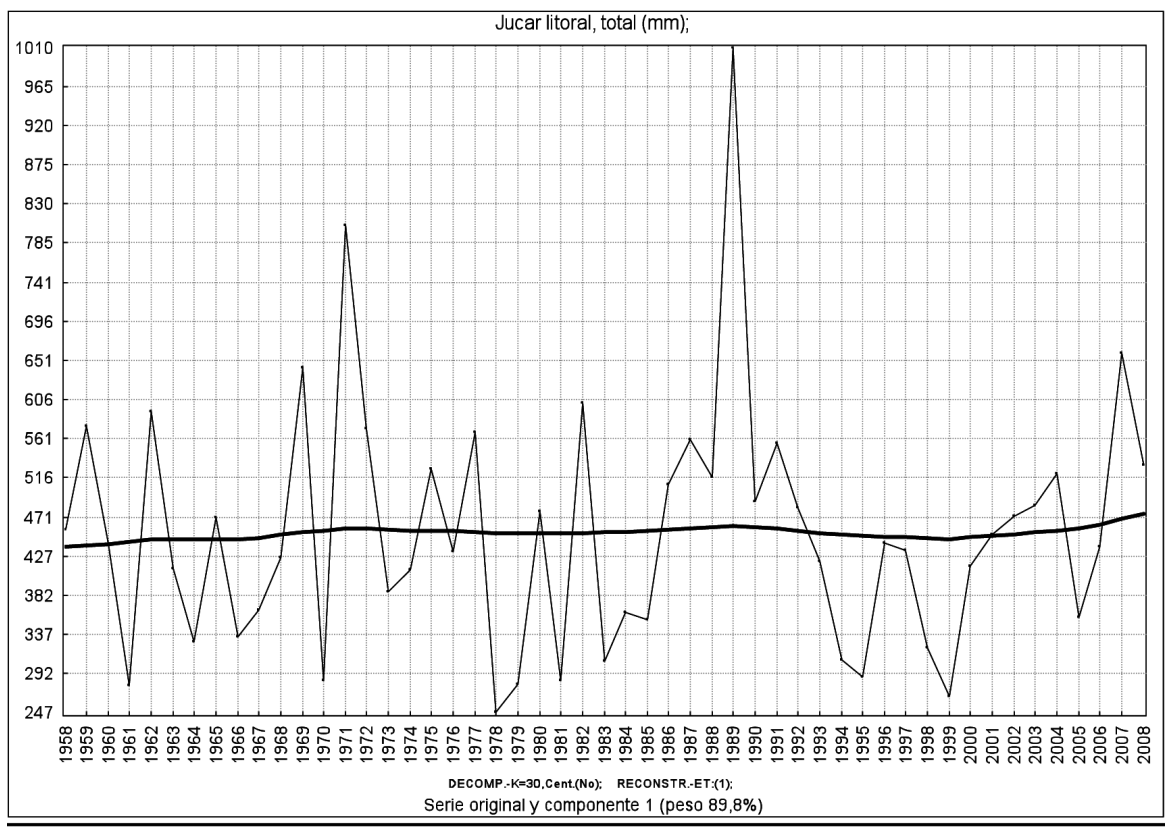

FIGURA 24. Precipitación total para el sector 'Júcar litoral' y tendencia extraída mediante SSA. 


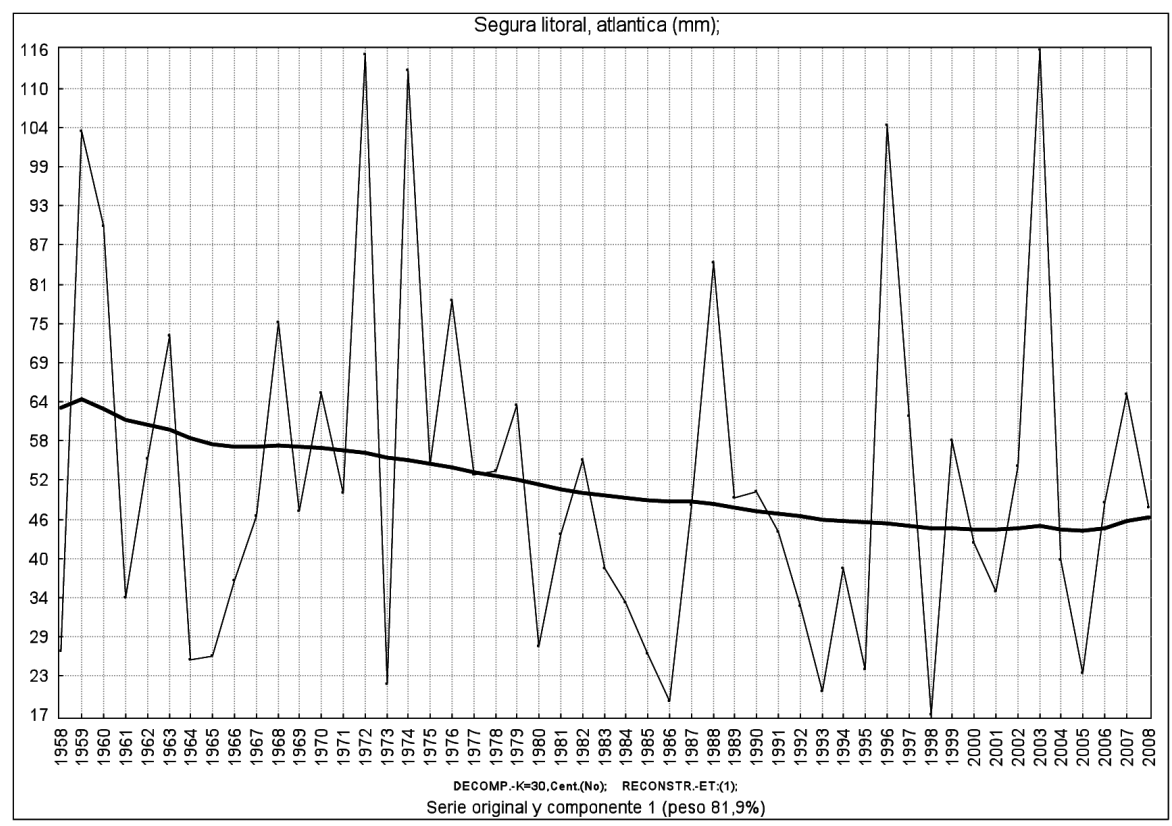

FIGURA 25. Precipitación por advección atlántica para el sector 'Segura litoral' y tendencia extraída mediante SSA.

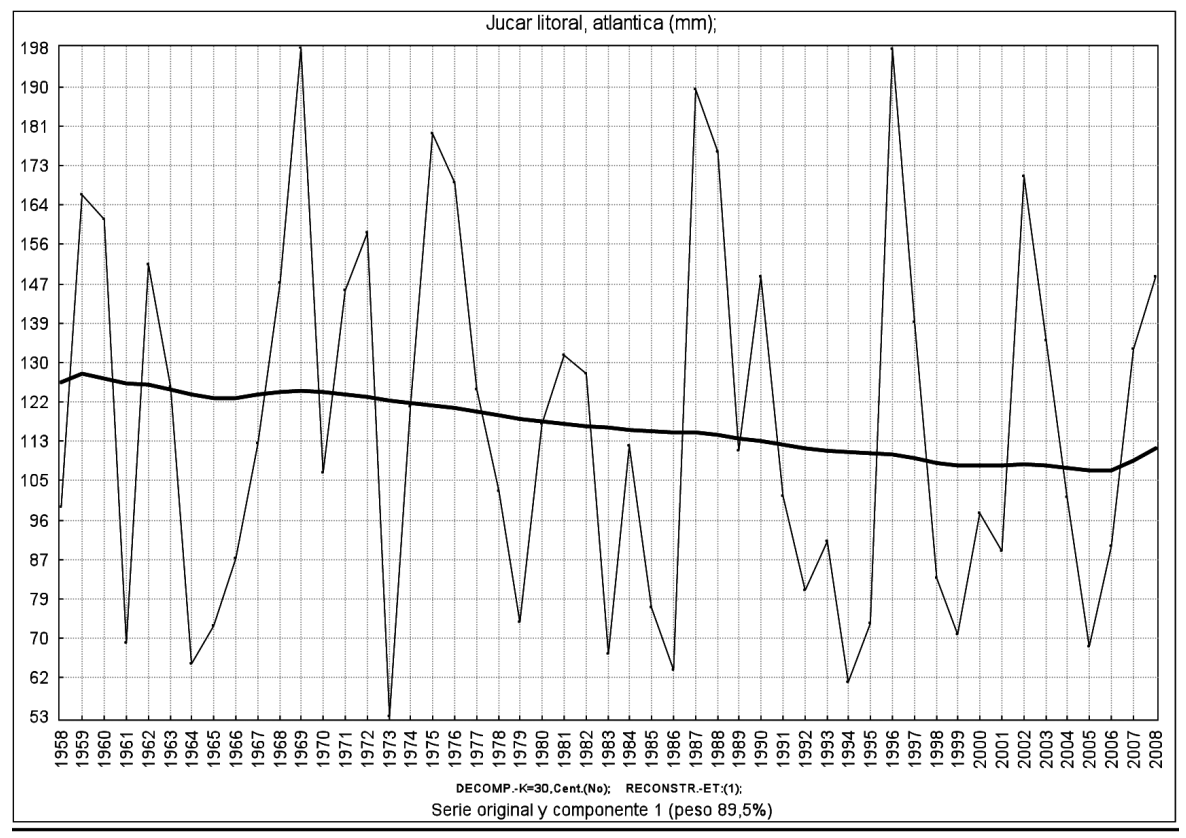

FIGURA 26. Precipitación por advección atlántica para el sector 'Júcar litoral' y tendencia extraída mediante SSA. 


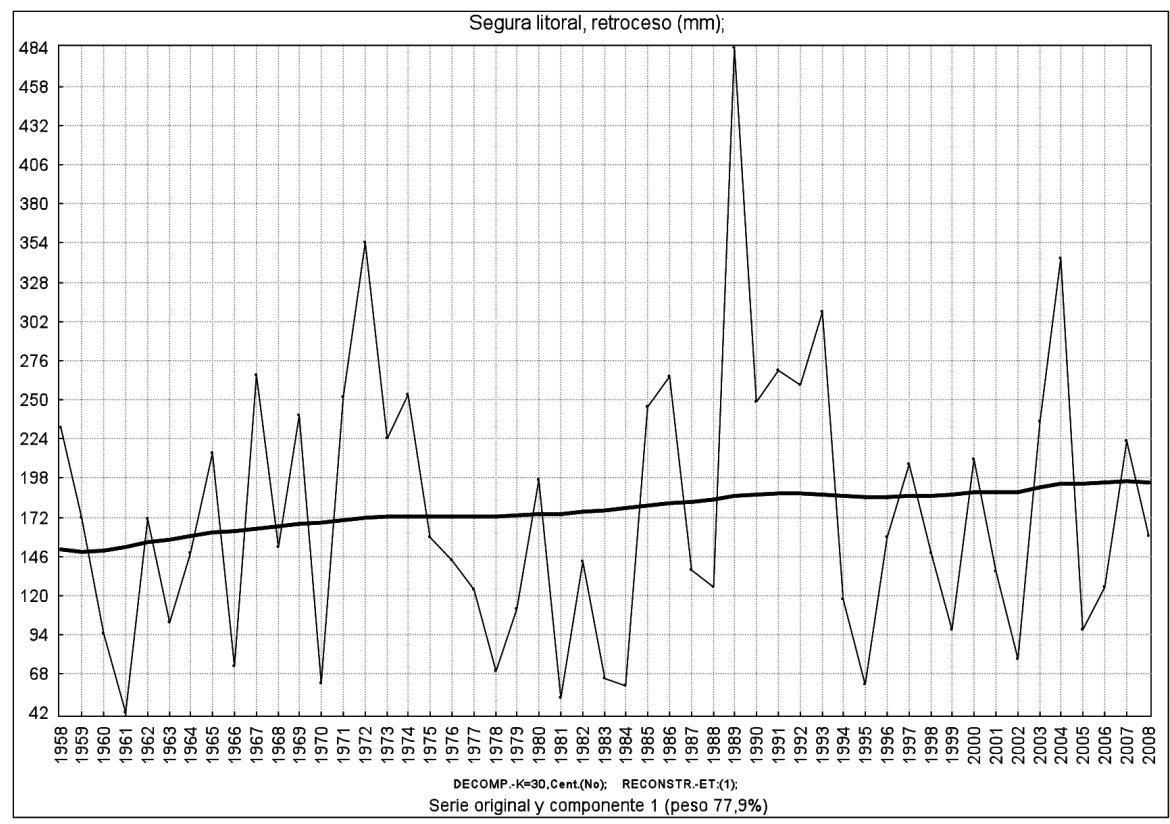

FiguRa 27. Precipitación por advección mediterránea, o de retroceso, para el sector 'Segura litoral' y tendencia extraída mediante SSA.

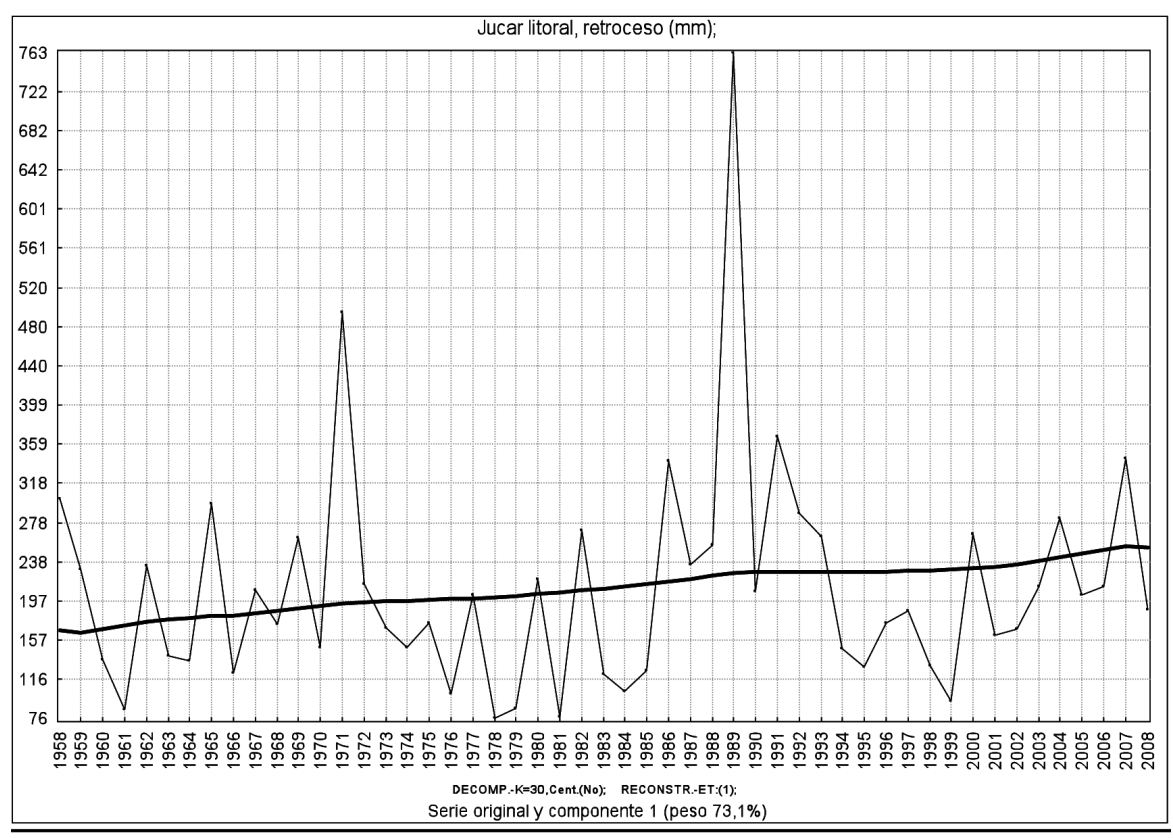

FIGURA 28. Precipitación por advección mediterránea, o de retroceso, para el sector 'Júcar litoral' y tendencia extraída mediante SSA. 


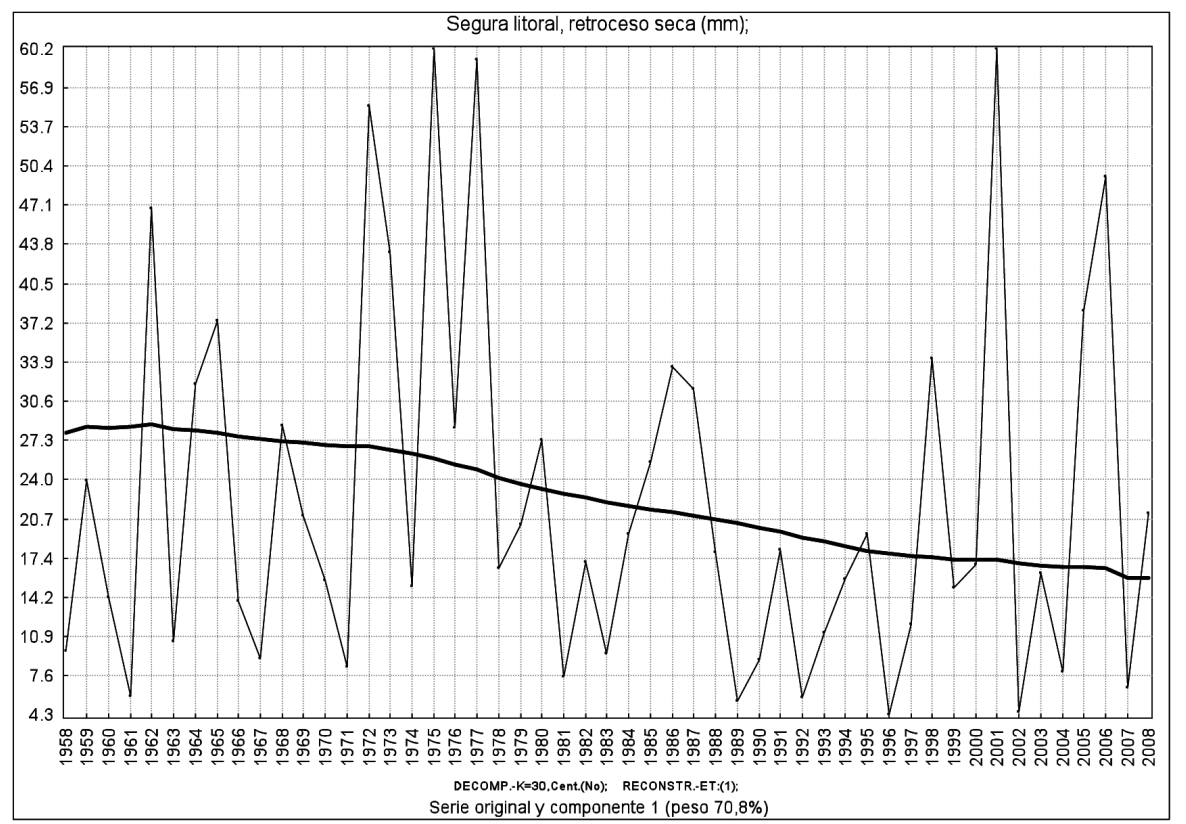

Figura 29. Precipitación por advección continental, o de retroceso seca, para el sector 'Segura litoral' y tendencia extraída mediante SSA.

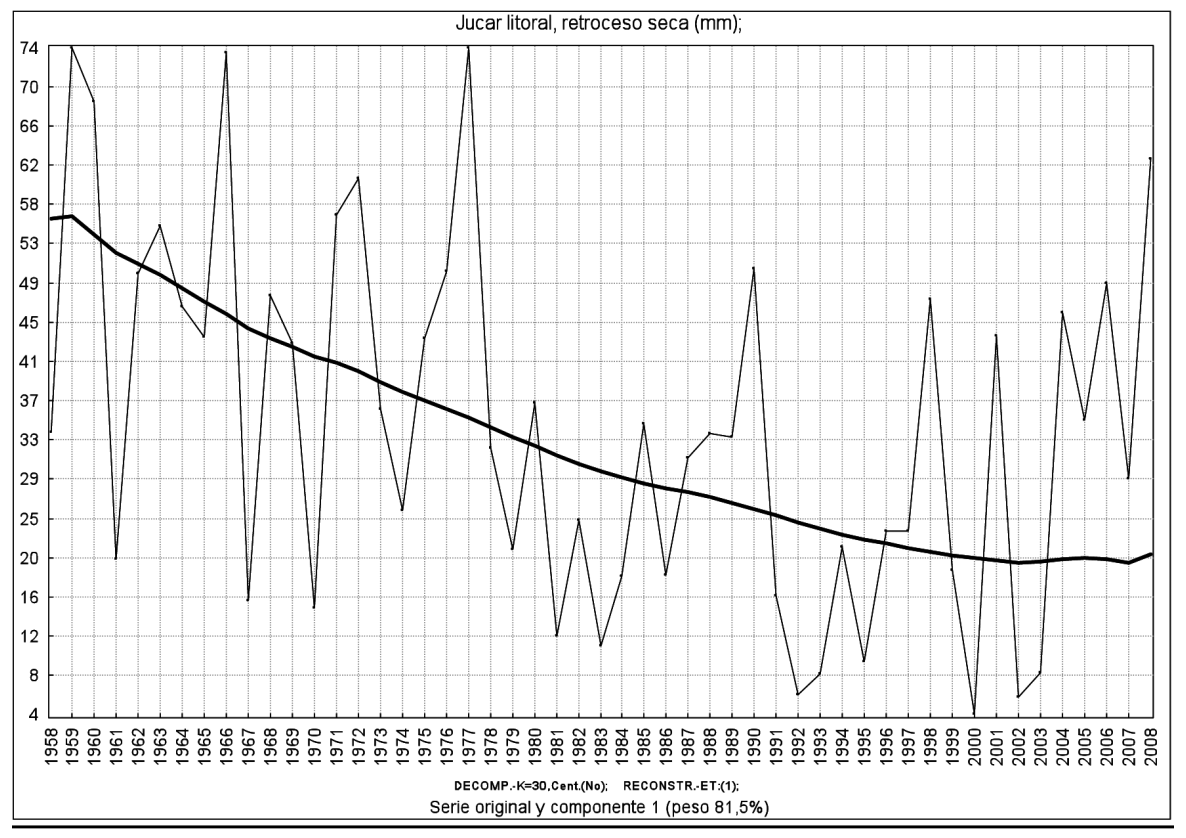

FIgURA 30. Precipitación por advección continental, o de retroceso seca, para el sector 'Júcar litoral' y tendencia extraída mediante SSA. 


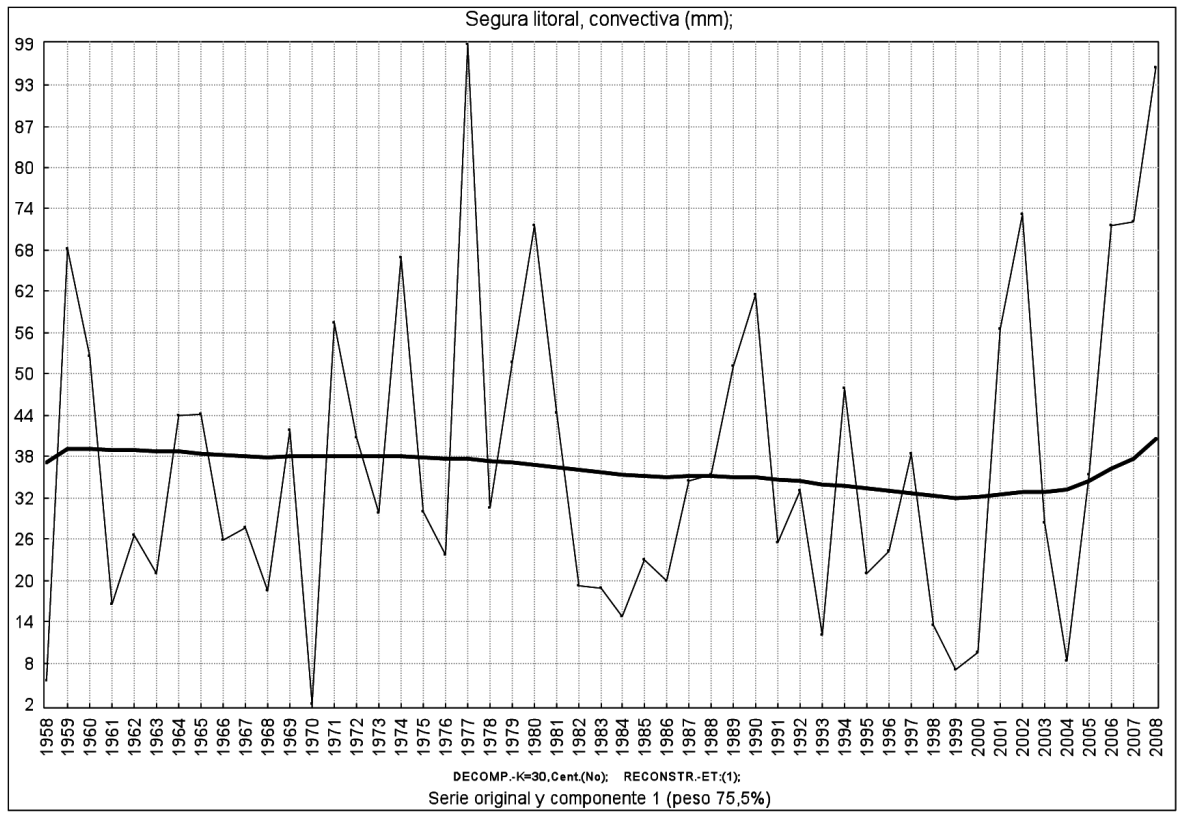

FIGURA 31. Precipitación por situaciones convectivas para el sector 'Segura litoral' y tendencia extraída mediante SSA.

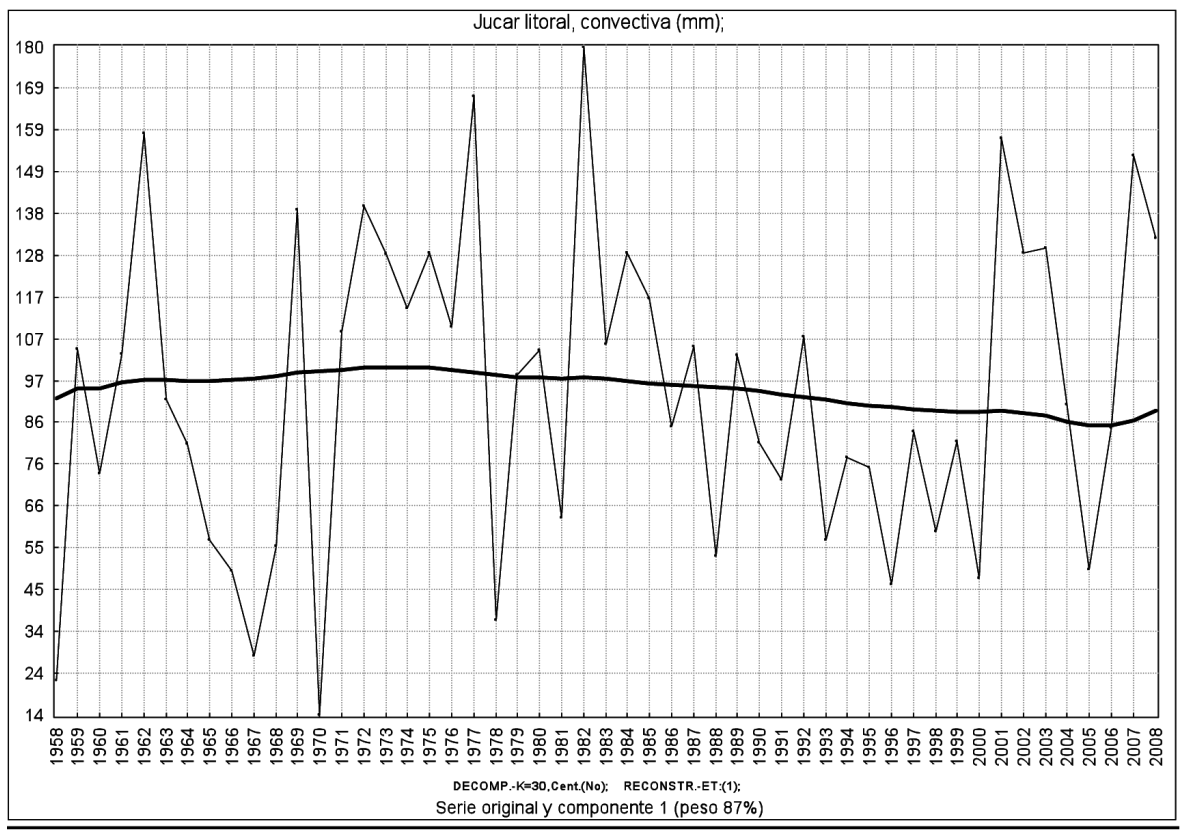

FIGURA 32. Precipitación por situaciones convectivas para el sector 'Júcar litoral' y tendencia extraída mediante SSA. 
Esta tendencia negativa para el input 'retroceso seca', también aparece en el sector 'Segura litoral', así como para el input 'atlánticas', tanto en el sector 'Jucar litoral' como en el de 'Segura litoral', todo ello en consonancia con lo observado en todo el resto de sectores. Para estos casos ya no se han obtenido buenos niveles de significación y confianza en los valores negativos que muestran el test de Mann-Kendall y pendiente Sen (Tabla 1). Sin embargo, la pendiente negativa se observa bien en la tendencia extraída mediante SSA (Figs. 25, 26 y 29).

El peso mayoritario del input 'retroceso' en estos dos sectores litorales compensa plenamente cualquier descenso experimentado por el resto de inputs, ya que en este caso se observa, tanto en el sector litoral del Segura como del Júcar, una suave pendiente al ascenso en la tendencia extraída por SSA (Figs. 27 y 28). En cualquier caso es dudosa en el test de Mann-Kendall y estimación Sen, aunque es concordante con la pauta de fondo observada en el resto de sectores.

Por último, para el caso de las 'convectivas', al igual que ocurre en la mayoría de sectores, las tendencias son inexistentes o poco definidas (Tabla 1, y Figs. 31 y 32).

\subsection{Cambios en el peso relativo de los distintos inputs pluviométricos}

A tenor de los resultados anteriores, parece evidente que las tendencias divergentes entre los distintos inputs se van a traducir en cambios en el peso relativo de cada input sobre el total anual de precipitación a lo largo del tiempo. Así, según las tendencias observadas parece que se va a producir una pérdida de protagonismo de las precipitaciones de origen atlántico y septentrional, a favor de las precipitaciones de origen mediterráneo. En este sentido, se ha calculado el porcentaje o peso de cada input en el volumen total de la precipitación, separando en dos grupos distintos los primeros años y los últimos de toda la serie utilizada (1958-2008). Así compararemos el grupo de años de 1958 a 1978 con el grupo de años de 1988 a 2008. Los resultados se muestran en las figuras 33 (interior), 34 (prelitoral) y 35 (litoral).

Puede observarse como, en todos los sectores sin excepción, se produce una pérdida significativa de los porcentajes de contribución de los inputs 'atlántica' y 'retroceso seca', a favor del input 'retroceso', que adquiere un importante ascenso. La precipitación de carácter convectivo generalmente se mantiene, o bien pierde algún punto porcentual.

En los sectores del interior la pérdida del peso relativo de las precipitaciones de origen atlántico ronda del 4 al 6\%, lo que es especialmente dramático para el área del 'Jucar interior', dado que es el único sector, de todos los analizados, en el que este input es el principal, con un peso en origen del $42 \%$, que desciende al $38 \%$. De hecho, esto explica lo ya visto con las tendencias negativas de este sector en el apartado anterior. Por otro lado, en ambos sectores interiores del Júcar y Segura la precipitación por advecciones mediterráneas ('retroceso') aumenta su contribución un $8 \%$, lo que a su vez supone aumentos entre el 20 y $30 \%$ en su mismo valor porcentual de partida. También supone un descenso considerable el salto del input 'retroceso seca', que pasa de tener un $7 \%$ de contribución a sólo un 4-5\% en ambas zonas.

Para los dos sectores prelitorales se repite la tendencia al ascenso del input 'retroceso', que suma entre 6 y 8 puntos porcentuales más, sobre el total, en el segundo periodo que en el primero. También se repite la pérdida del input 'atlánticas', en este caso de 3 a 4 puntos porcentuales, lo que, dado el menor peso relativo de partida, supone un descenso de una magnitud similar al ascenso experimentado por el input 'retroceso'. Para el caso de las precipitaciones de origen 'retroceso seca', aunque tenga un peso bajo de partida, el paso de una contribución del 9-10\% a una contribución del 6\% supone una disminución muy importante. 


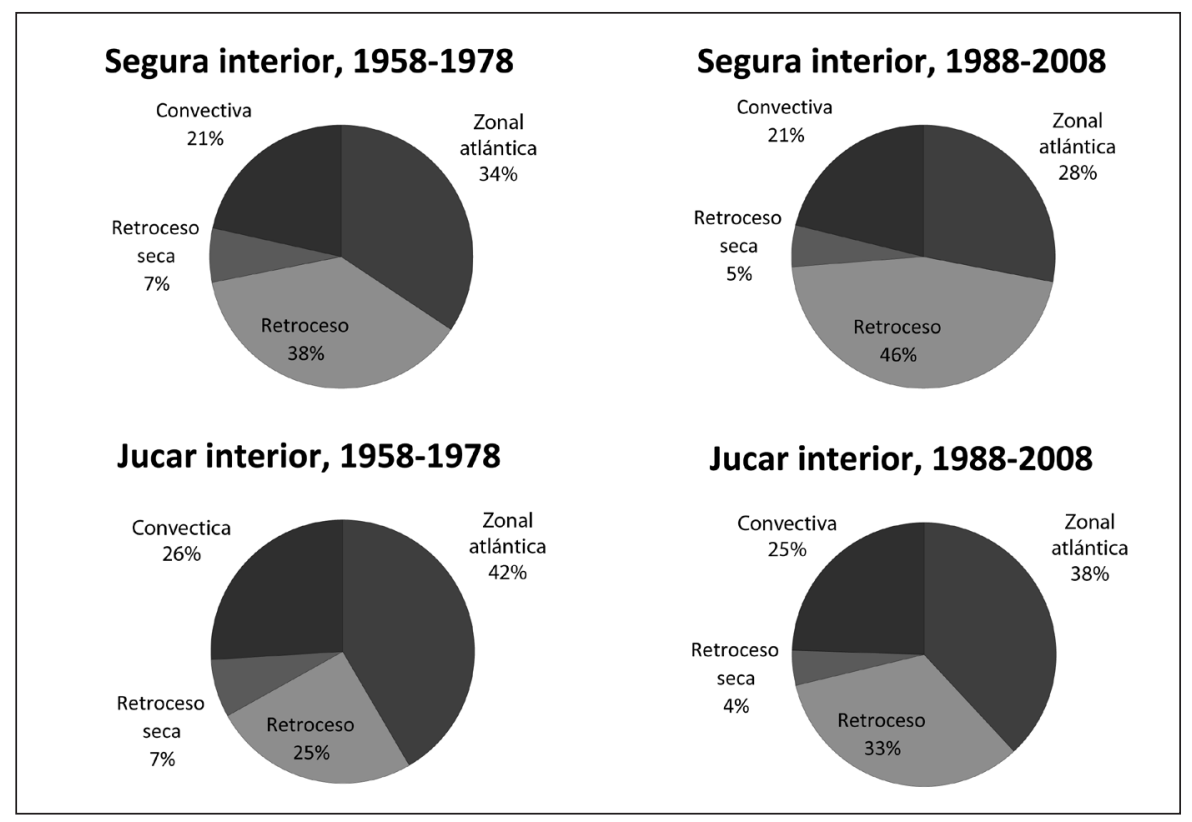

FIgURA 33. Sectores del interior. Distribución del porcentaje que representa cada input sobre el total de precipitación anual. A) Período 1958-1978. B) Período 1978-2008.

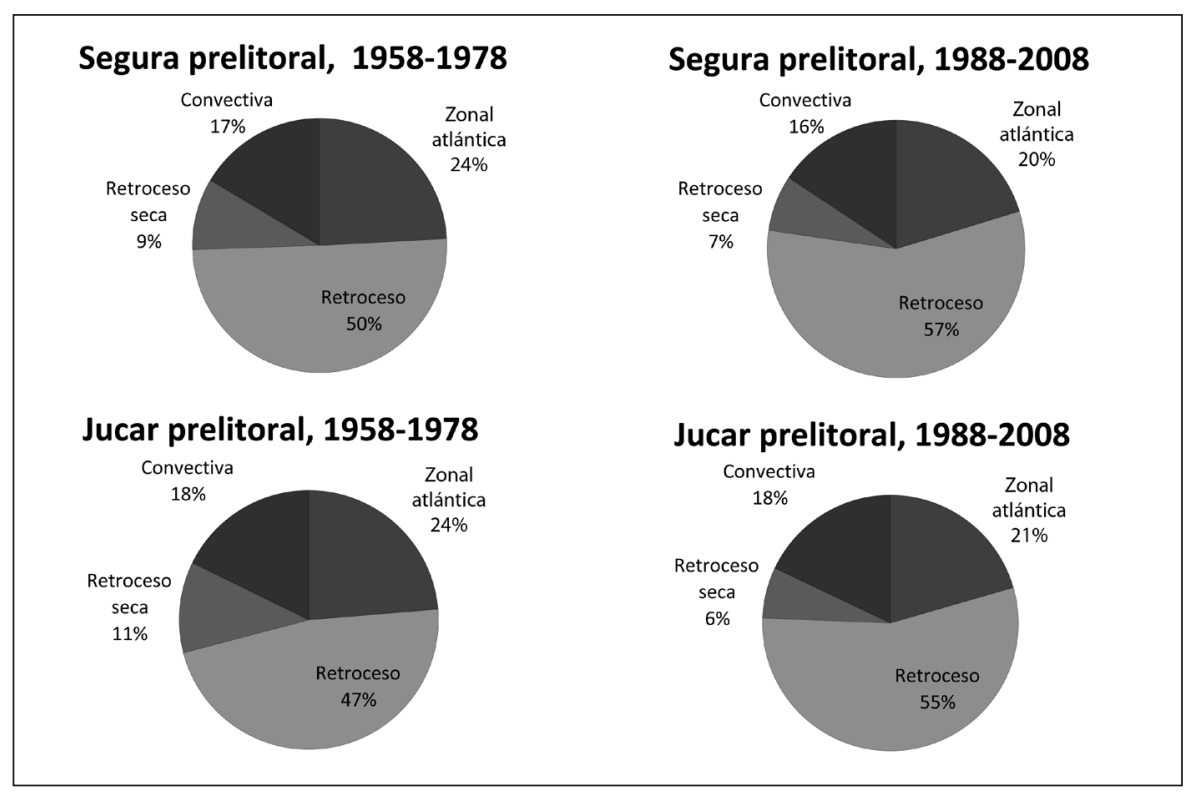

FIgURA 34. Sectores del prelitoral. Distribución del porcentaje que representa cada input sobre el total de precipitación anual. A) Período 1958-1978. B) Período 1978-2008. 


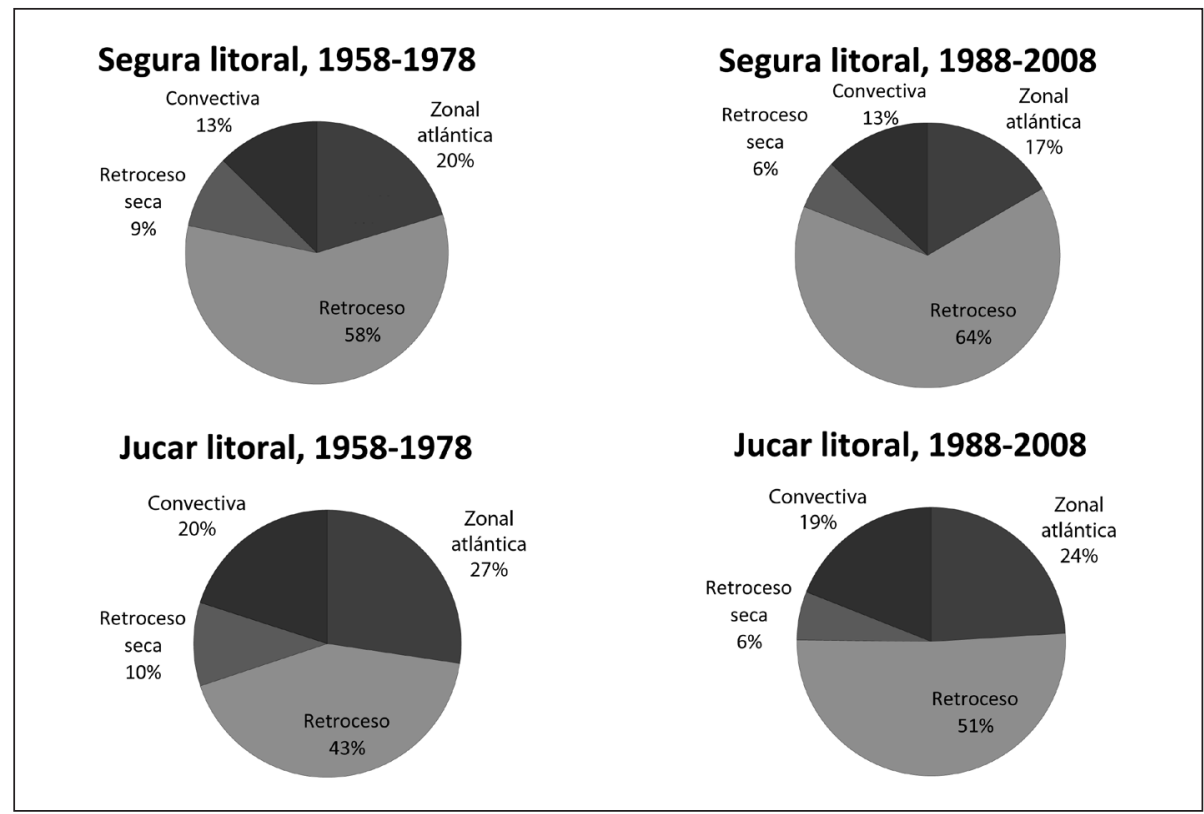

FIGURA 35. Sectores del litoral. Distribución del porcentaje que representa cada input sobre el total de precipitación anual. A) Período 1958-1978. B) Período 1978-2008.

Respecto de los sectores litorales, se reproduce lo señalado para los prelitorales, repitiéndose la significativa pérdida de peso de los inputs 'atlántica' y 'retroceso seca', a favor del input 'retroceso'.

\section{Conclusiones}

A partir del análisis realizado, se confirma la existencia de un cambio estructural en la precipitación en los últimos 50 años.

En primer lugar, aunque es cierto que en los totales anuales no hay tendencias significativas en la mayoría de sectores analizados (al menos en los sectores litorales), a partir del estudio desagregado de la precipitación por inputs sí se detectan tendencias significativas. Estas son más notables e indican sobre todo un descenso en el caso de las advecciones atlánticas y las septentrionales para los dos dominios hidrológicos analizados. Sin embargo, el peso mayor que el input 'atlántico' tiene en el interior Norte, ha favorecido que sea sólo en el sector 'Júcar interior' donde realmente esta disminución está teniendo un impacto notable, incluso también detectable en los totales anuales de precipitación. Tendencias al descenso muy acusadas también se aprecian en las advecciones septentrionales o de 'retroceso seca', aunque este input en ningún caso tiene una contribución al total importante.

Frente a la disminución, volumétricamente significativa de las precipitaciones de origen 'atlántico', toman el relevo de éstas las precipitaciones de origen mediterráneo que, o bien se mantienen, o bien incluso hay indicios de que aumentan en valor absoluto en algún caso, como ocurre en el sector 'Júcar prelitoral', donde incluso el total anual muestra una suave, en todo caso dudosa, tendencia al ascenso. 
A partir de los datos se puede afirmar que ha habido un cambio, en todos los sectores y para los dos dominios hidrológicos, en la propia distribución de contribuciones a la precipitación total de los distintos inputs. La aportación desde el Mediterráneo le está ganando porcentaje a todo el resto de inputs, especialmente al de 'atlánticas' y 'retroceso seca'. Dado el carácter propio de la precipitación por advecciones mediterráneas, más irregular, concentrada en el tiempo y de mayor variabilidad por la alternancia con períodos secos, se puede concluir que en los últimos 50 años se ha asistido a una acentuación del carácter 'mediterráneo' de la precipitación en ambos dominios hidrológicos. Ello va en apoyo de otros estudios que, en esta misma línea, están confirmando una tendencia al aumento de la irregularidad de la precipitación (Almarza, 2002).

\section{Agradecimientos}

Este trabajo ha sido financiado por el Ministerio de Educación y Ciencia a través de los proyectos GRACCIE (Programa CONSOLIDER-INGENIO 2010 CSD2007-00067), NIEVA (CGL2008-04550), y por la Comisión Europea mediante el proyecto integrado CIRCE (Proj. No. 036931-2). La Fundación CEAM está financiada por la Generalitat Valenciana y Fundación Bancaja.

\section{Bibliografía}

ALEXANDROV, T. (2006): «Software package for automatic extraction and forecast of additive components of time series in the framework of the Caterpillar-SSA approach». PhD thesis, St. Petersburg State University.

ALEXANDROV, T.; GOLYANDINA, N. (2005): «Thresholds for methods of automatic extraction of time series trend and periodical components with the help of the «Caterpillar»-SSA approach». In: Proc. 4th Conf. System 8 Identification and Control Problems. Inst. of Control Sci, pp. 1849-1864, Moscow.

ALMARZA, C. (2002): «La estructura de la precipitación como índice de detección de cambio climático». Asamblea de Geodesia y Geofísica de Valencia. 4-8 Febrero 2002. Instituto Nacional de Meteorología, Madrid.

CHAZARRA, A.; ALMARZA, C. (2002): «Reconstrucción desde 1864 de la serie de precipitación útil de las cuencas del Sureste y Levante». En: GUIJARO, J.A.; Editor Springer Wien, Volume 71, Numbers 1-2, pp. 77-95.

ELSNER, J. B.; TSONIS, A. A. (1996): «Singular Spectral Analysis. A New Tool in Time Series Analysis». Plenum Press.

ESTEBAN-PARRA, M. J.; POZO-VÁZQUEZ, F. S.; RODRIGO, CASTRO DIEZ, Y. (1998): «Singular Spectral Analysis of the Spanish Precipitation». In Proceedings «14th Conference on Statistic in the Atmospheric Sciences». American Meteorological Society, pp. 103-106, U.S.A. ISBN: D(VT)1/98-400.

ESTEBAN-PARRA, M. J.; POZO-VÁZQUEZ, F. S.; RODRIGO, CASTRO DIEZ, Y. (1999): «Análisis espectral singular de las series de temperatura en España. / Singular spectral analysis of the temperature series in Spain». En: I Asamblea Hispano Portuguesa de Geodesia y Geofísica. Universidad de Almería. Instituto Geográfico Nacional, España, CD-ROM, ISBN: 84-95172-10-0.

ESTRELA, M. J.; MIRÓ, J. J.; MILLAN, M. (2006): «Análisis de tendencia de la precipitación por situaciones convectivas en la Comunidad Valenciana (1959-2004)». En: Clima, Sociedad y Medio Ambiente. (Cuadrat, J. M., Saz, M. A., Vicente, S. M., Lanjeri, S., De Luis, M., and GonzálezHidalgo, J. C., eds.), pp. 125-136. Asociación Española de Climatología. Zaragoza.

ESTRELA, M. J.; MIRÓ, J. J.; PASTOR, F.; MILLÁN, M. (2004): «Precipitaciones por frentes atlánticos en la Comunidad Valenciana: cambios y tendencias en las últimas décadas». En: XXVIII Jornadas Científicas de la Asociación Meteorológica Española. 11-13 Febrero 2004. Badajoz.

ESTRELA, M. J.; PEÑARROCHA, D.; MILLÁN, M. (2000): «Multi-annual drought episodes in the mediterranean (Valencia Region) from 1950-1996. A spatio-temporal analysis». International Journal of Climatology, 20, pp. 1599-1618. 
GARCÍA, J. A.; SERRANO, A.; CRUZ GALLEGO, M. (2002): «A spectral analysis of Iberian Peninsula monthly rainfall». Theoretical and Applied Climatology.

HOUGHTON, J. T.; MEIRA FILHO, L. G.; CALLANDER, B. A.; HARRIS, N.; KATTENBERG, A.; MASKELL, K. (Eds.) (1996): «IPCC Climate Change. The IPCC Second Assessment Report». Cambridge University Press, 572 pp. New York.

KENDALL, M. G. (1975): «Rank correlation methods» $4^{\text {th }}$ Ed., Charles Griffin, Londres.

MANN, H. B. (1945): «Non-parametric test against trend». Econometrica, 13, pp. 245-249.

MARTÍN-VIDE, J. (1987): «Característiques climatologiques de la precipitació en la franja costera mediterrania de la Peninsula Ibérica». Institut Cartografic de Catalunya. Generalitat de Catalunya, 245 pp. Barcelona.

MILLÁN, M.; ARTIÑANO, B. (1992): «Mesometeorological cycles of air pollution in the Iberian Peninsula». Brussels: Commission of the European Communities. 219 pp.

MILLÁN, M.; ESTRELA, M. J.; MIRÓ, J. J. (2005): «Rainfall components: variability and spatial distribution in a mediterranean area (Valencia Region)». Journal of Climate, 18 (14), pp. $2682-$ 2705.

MILLÁN, M. M.; ESTRELA, M. J.; MIRÓ, J. J. (2006): «Análisis de tendencia de la precipitación bajo situaciones de frente de retroceso en la Comunidad Valenciana (1959-2004)». En: Clima, Sociedad y Medio Ambiente. (Cuadrat, J. M., Saz, M. A., Vicente, S. M., Lanjeri, S., De Luis, M., and González-Hidalgo, J. C., eds.), pp. 199-209. Asociación Española de Climatología. Zaragoza.

MIRÓ, J. J.; ESTRELA, M. J.; MILLÁN, M. M. (2006): «Análisis de tendencia de la precipitación por frentes atlánticos en la Comunidad Valenciana (1959-2004)». En: Clima, Sociedad y Medio Ambiente. (Cuadrat, J. M., Saz, M. A., Vicente, S. M., Lanjeri, S., De Luis, M., and GonzálezHidalgo, J. C., eds.), pp. 211-220. Asociación Española de Climatología. Zaragoza.

MONTÓN, E.; QUEREDA, J. (1997): «¿Hacia un cambio climático? La evolución del clima mediterráneo desde el siglo XIX». Fundación Davalos-Fletcher, 520 pp. Valencia.

RASO NADAL, J. M. (1996): «Variación de las precipitaciones de primavera en el sur de la España peninsular durante el siglo XX». En: MARZOL, M. V.; DORTA; P.; VALLADARES, P. (Eds.). Clima y agua: la gestión de un recurso climático, pp. 123-132. La Laguna.

RODRÍGUEZ-PUEBLA, C.; ENCINAS, A. H.; NIETO, S.; GARMENDÍA, J. (1998): «Spatial and temporal patterns of annual precipitation variability over the Iberian peninsula». Int. J. of Climatology, 18, 299-316.

SALES MARTÍNEZ, V.; JAMBRINO CALVET, T.; JUSTE PÉREZ, J. J. (1982): «Análisis espacial y temporal de la sequía 1978-1981 en España». Cuadernos de Geografía, 30, pp. 13-24.

SALMI, T.; MÄ̈̈TTÄ, A.; ANTTILA, P.; RUOHO-AIROLA, T.; AMNELL, T. (2002): «Makesens for detecting and estimating trends»: http://www.fmi.fi/organization/contacts_25.html

SCHÖNWIESE, C. D.; RAPP, J. (1997): «Climate trend atlas of Europe based on observations 18911990». Kluwer Academic Publishers, 224 pp. Dordrecht.

SEN, P. K. (1968): «Estimates of the regression coefficient based on Kendall's tau». Journal of the American Statistical Association. 63:1379-1389.

VAUTARD, R.; GHIL, M. (1989): «Singular spectrum analysis in nonlinear dynamics, with applications to paleoclimatic time series», Physica D, 35, 395-424. 
